\title{
A NEW ALGORITHM FOR STATE-CONSTRAINED SEPARATED CONTINUOUS LINEAR PROGRAMS*
}

\author{
XIAODONG LUO $^{\dagger}$ AND DIMITRIS BERTSIMAS $\ddagger$
}

\begin{abstract}
During the last few decades, significant progress has been made in solving largescale finite-dimensional and semi-infinite linear programming problems. In contrast, little progress has been made in solving linear programs in infinite-dimensional spaces despite their importance as models in manufacturing and communication systems. Inspired by the research on separated continuous linear programs, we propose a new class of continuous linear programming problems that has a variety of important applications in communications, manufacturing, and urban traffic control. This class of continuous linear programs contains the separated continuous linear programs as a subclass. Using ideas from quadratic programming, we propose an efficient algorithm for solving large-scale problems in this new class under mild assumptions on the form of the problem data. We prove algorithmically the absence of a duality gap for this class of problems without any boundedness assumptions on the solution set. We show this class of problems admits piecewise constant optimal control when the optimal solution exists. We give conditions for the existence of an optimal solution. We also report computational results which illustrate that the new algorithm is effective in solving large-scale realistic problems (with several hundred continuous variables) arising in manufacturing systems.
\end{abstract}

Key words. continuous linear programming, strong duality, semi-infinite linear programming, nonlinear programming, jobshop scheduling problems

AMS subject classifications. 49J15, 49K30, 49M35, 49M37, 49M39, 90C34, 90C35

PII. S0363012995292664

1. Introduction. Bellman $[7,8]$ introduced the following optimization problem:

$$
\begin{aligned}
& (C L P) \text { minimize } \int_{0}^{T} c(t)^{\prime} x(t) d t \\
& \text { subject to (s.t.) } A(t) x(t)+\int_{0}^{t} B(s, t) x(s) d s \leq b(t), \\
& \qquad x(t) \geq 0, \quad t \in[0, T],
\end{aligned}
$$

where $A(t)$ and $B(s, t)$ are matrices depending on time (their entries are bounded measurable functions) and $b(t)$ and $c(t)$ are bounded measurable functions. $(C L P)$ is an instance of a continuous linear program.

The problem that has attracted the most attention is the separated continuous linear programming problem (SCLP), a subclass of the continuous linear programming problem:

$$
(S C L P) \text { minimize } \int_{0}^{T} c(t)^{\prime} u(t) d t
$$

*Received by the editors October 4, 1995; accepted for publication (in revised form) February 9, 1998; published electronically October 7, 1998.

http://www.siam.org/journals/sicon/37-1/29266.html

†Operations Research Center, Massachusetts Institute of Technology, Cambridge, MA 02139 (xluo@ athena.mit.edu).

${ }^{\ddagger}$ Sloan School of Management, Massachusetts Institute of Technology, 50 Memorial Dr., Room E53-363, Cambridge, MA 02139 (dbertsim@mit.edu). The research of the author was partially supported by Presidential Young Investigator Award DDM-9158118 with matching funds from Draper Laboratory and NSF grant DMI-9610486. 


$$
\begin{aligned}
& \text { s.t. } \int_{0}^{t} G u(t) d t+y(t)=a(t), \\
& H u(t) \leq b(t), \\
& y(t), u(t) \geq 0, \quad t \in[0, T],
\end{aligned}
$$

where $y(t)$ and $a(t)$ are absolutely continuous functions. Note that the variables $u(t)$ and $y(t)$ are linked only through (1), in which $u(t)$ appears only under the integration operator and $y(t)$ does not appear under the integration operator. The problem $(S C L P)$ was first introduced by Anderson [4] in order to model job-shop scheduling problems (see also Avram, Bertsimas, and Ricard [6], Weiss [49]).

In this paper, we examine a larger subclass of continuous linear programs which can be used to model a variety of problems that arise in communications, manufacturing, and urban traffic control (see Luo [32]). The problem we consider is the following:

$$
\begin{array}{cl}
(S C S C L P) \text { minimize } & \int_{0}^{T}\left(c(t)^{\prime} u(t)+g(t)^{\prime} y(t)\right) d t \\
\text { s.t. } & \int_{0}^{t} G u(t) d t+E y(t)=a(t), \\
& H u(t) \leq b(t), \\
& F y(t) \leq h(t), \\
& u(t) \geq 0, \quad t \in[0, T],
\end{array}
$$

where $b(t), c(t), g(t)$, and $h(t)$ are bounded measurable functions and $a(t)$ is an absolutely continuous function. The dimensions of $b(t), a(t), u(t), y(t)$, and $h(t)$ are $n_{1}, n_{2}, n_{3}, n_{4}$, and $n_{5}$, respectively. We call $(S C S C L P)$ the state-constrained separated continuous linear programs. We call $y(t)$ the state variable and $u(t)$ the control variable. We call (2) the state equation (or sometimes we use the term system dynamics) and call (4) the state constraint. We call (3) the control constraint.

Related literature. The computational study of CLP was initiated by Lehman [26] who attempted to develop a simplex-like algorithm for CLP. Drews [10], Hartberger [20], and Segers [44] later followed him. Perold [37, 38] developed the first simplexlike algorithm for CLP (see also Anderson, Nash, and Perold [1] and Anderson and Philpott [3]. Anstreicher [5] continued Perold's work in his Ph.D. thesis, even though both their algorithms were still incomplete. In the meantime, Russian authors such as Ilyutovich $[21,22]$ treated the problem using Pontryagin's maximum principle. In addition, Ito, Kelley, and Sachs [23] have developed a primal-dual path, following interior point method for CLP. Anderson and Nash in [2] proposed a convex quadratic programming procedure for $(S C L P)$. The series of papers on SCLP by Pullan [41, $42,43]$ deals with solution structure, duality theory, and numerical algorithms and to the best of our knowledge represents the state of the art of this area. Philpott and Craddock [39] later specialized Pullan's work to a network version of SCLP and presented encouraging numerical results.

Objective and contributions. In this paper, we will develop a new algorithm for solving SCSCLP problems under Assumption 1 below. The new algorithm uses discretization. Unlike the algorithms mentioned above, it varies the discretization and control simultaneously. Based on the number of constant pieces allowed in the control, we develop a quadratic program with polyhedral constraints. The quadratic 
program is generally nonconvex. However, we do not need to solve the quadratic program to optimality. We only need to obtain a KKT point. We use the Frank-Wolfe method (see Martos [33] and Murty [36]) or general matrix-splitting algorithms (see Lin and Pang [28], Eckstein [11], Bertsekas and Tsitsiklis [9], Luo and Tseng [31]) to find a KKT point for the quadratic program. By gradually increasing (and occasionally decreasing) the number of pieces allowed in the control, we can improve upon any nonoptimal KKT solution. We call this the successive quadratic programming method. By a KKT solution structural result of Luo and Tseng [31], we show that the iterates of the algorithm move from one polyhedral set to another, with improved cost. By bounding the size of the quadratic programs we encounter, we bound the number of all such polyhedral sets. We show that the new algorithm converges in finite time. The absence of a duality gap and the existence of certain highly structured optimal solutions for $(S C S C L P)$ follow as byproducts. Furthermore, we have implemented our algorithm and report computational results which illustrate that the new algorithm is effective in solving large scale realistic problems (with several hundred continuous variables) arising in manufacturing systems.

Structure of the paper. The remainder of this paper is structured as follows. In section 2, we introduce the dual problem for $(S C S C L P)$ (called $\left(S C S C L P^{*}\right)$ ) and state our assumptions. We also prove weak duality results between $(S C S C L P)$ and $\left(S C S C L P^{*}\right)$ and introduce some standard definitions and notations. In section 3 , we develop a quadratic program with polyhedral constraints. In section 3.1 , we review some nonlinear programming techniques for calculating a KKT point of a quadratic program with polyhedral constraints. In section 4, we develop a procedure for removing redundant intervals in a feasible solution for $(S C S C L P)$. In section 5, we introduce a new discrete approximation for $(S C S C L P)$ which is closely related to the dual problem. From this discrete approximation, we derive a criterion to detect whether a feasible solution is optimal for $(S C S C L P)$. If the criterion is not satisfied, we derive a descent direction for the feasible solution to $(S C S C L P)$. In section 6 , we formally state the new algorithm. In section 7 , we prove that the new algorithm converges in finite time. In section 8 , we use the new algorithm to prove new duality results and new optimal solution structural results for $(S C S C L P)$. In section 9, we report computational results that illustrate the effectiveness of the new algorithm in solving large-scale problems. The reader is advised to first read sections 2 and 6 to obtain a general idea of the problem, the assumptions, and the new algorithm.

2. Definitions and notation. First, we reiterate problem (SCSCLP) and state our assumptions. We consider the problem

$$
\begin{aligned}
(S C S C L P) \text { minimize } & \int_{0}^{T}\left(c(t)^{\prime} u(t)+g(t)^{\prime} y(t)\right) d t \\
\text { s.t. } & \int_{0}^{t} G u(t) d t+E y(t)=a(t), \\
& H u(t) \leq b(t), \\
& F y(t) \leq h(t), \\
& u(t) \geq 0, \quad t \in[0, T],
\end{aligned}
$$

and its dual

$$
\left(S C S C L P^{*}\right) \text { maximize }-\int_{0}^{T} a(t)^{\prime} d \pi(t)-\int_{0}^{T} b(t)^{\prime} \eta(t) d t-\int_{0}^{T} h(t)^{\prime} d \xi(t)
$$




$$
\begin{array}{ll}
\text { s.t. } & c(t)-G^{\prime} \pi(t) d t+H^{\prime} \eta(t) \geq 0, \\
& E^{\prime} \pi(t)+F^{\prime} \xi(t)=\int_{t}^{T} g(t) d t \\
& \pi(t) \text { bounded measurable with finite variation, } \\
& \xi(t) \text { monotonic increasing and right continuous } \\
& \text { on }[0, T] \text { with } \xi(T)=0, \quad \pi(T)=0, \\
& \eta(t) \geq 0, \quad \text { for } t \in[0, T],
\end{array}
$$

under the following assumptions (we will give a formal definition of piecewise linear (constant) functions later in this section).

Assumption 1.

a) $a(t)$ and $h(t)$ are continuous,

b) $a(t), c(t)$, and $h(t)$ are piecewise linear,

c) $b(t)$ and $g(t)$ are piecewise constant,

d) Problem (SCSCLP) is feasible and its objective value is bounded from below.

We require that $u(t), y(t)$, and $\eta(t)$ are bounded and measurable functions on $[0, T]$. We remark that the dual problem $\left(S C S C L P^{*}\right)$ reduces to the alternative dual problem for $(S C L P)$ introduced by Pullan [41] when the primal problem is an SCLP.

We have the following weak duality results for $(S C S C L P)$. For completeness, we give its proof.

Proposition 1. Weak duality holds between (SCSCLP) and (SCSCLP*).

Proof. Consider any two solutions $(u(t), y(t))$ and $(\pi(t), \eta(t), \xi(t))$ which are feasible to $(S C S C L P)$ and $\left(S C S C L P^{*}\right)$, respectively. Let $z(t)=b(t)-H u(t)$ and $\bar{z}(t)=h(t)-F y(t)$. We have

$$
\begin{aligned}
\int_{0}^{T}\left(c(t)^{\prime} u(t)+g(t)^{\prime} y(t)\right) d t-\left(-\int_{0}^{T} a(t)^{\prime} d \pi(t)-\int_{0}^{T} b(t)^{\prime} \eta(t) d t-\int_{0}^{T} h(t)^{\prime} d \xi(t)\right) \\
=\int_{0}^{T}\left(c(t)^{\prime} u(t)+g(t)^{\prime} y(t)\right) d t+\int_{0}^{T} a(t)^{\prime} d \pi(t)+\int_{0}^{T} b(t)^{\prime} \eta(t) d t+\int_{0}^{T} h(t)^{\prime} d \xi(t) \\
=\int_{0}^{T}\left(c(t)^{\prime} u(t)+g(t)^{\prime} y(t)\right) d t+\int_{0}^{T}\left(\int_{0}^{t} G u(s) d s+E y(t)\right)^{\prime} d \pi(t) \\
\quad+\int_{0}^{T}(H u(t)+z(t))^{\prime} \eta(t) d t+\int_{0}^{T}(F y(t)+\bar{z}(t))^{\prime} d \xi(t) \\
=\int_{0}^{T}\left(c(t)^{\prime} u(t)+g(t)^{\prime} y(t)\right) d t-\int_{0}^{T} \pi(t)^{\prime} G u(t) d t+\int_{0}^{T}(E y(t))^{\prime} d \pi(t) \\
\quad+\int_{0}^{T}(H u(t)+z(t))^{\prime} \eta(t) d t+\int_{0}^{T}(F y(t)+\bar{z}(t))^{\prime} d \xi(t) \\
=\int_{0}^{T}\left(c(t)-G^{\prime} \pi(t)+H^{\prime} \eta(t)\right)^{\prime} u(t) d t \\
\quad+\int_{0}^{T} y(t)^{\prime} d\left(E^{\prime} \pi(t)+F^{\prime} \xi(t)-\int_{t}^{T} g(t) d t\right) \\
\quad+\int_{0}^{T} z(t)^{\prime} \eta(t) d t+\int_{0}^{T} \bar{z}(t)^{\prime} d \xi(t)
\end{aligned}
$$


$=\int_{0}^{T}\left(c(t)-G^{\prime} \pi(t)+H^{\prime} \eta(t)\right)^{\prime} u(t) d t+\int_{0}^{T} z(t)^{\prime} \eta(t) d t+\int_{0}^{T} \bar{z}(t)^{\prime} d \xi(t)$

$\geq 0$.

Note that in general $\int_{0}^{T}(F y(t))^{\prime} d \xi(t)$ and $\int_{0}^{T} \bar{z}(t)^{\prime} d \xi(t)$ may not exist since neither $y(t)$ nor $\bar{z}(t)$ needs to be continuous. However, since

$$
\int_{0}^{T} y(t)^{\prime} d\left(E^{\prime} \pi(t)+F^{\prime} \xi(t)-\int_{t}^{T} g(t) d t\right)=0,
$$

we have

$$
\begin{aligned}
\int_{0}^{T} y(t)^{\prime} d F^{\prime} \xi(t) & =\int_{0}^{T} y(t)^{\prime} d\left(E^{\prime} \pi(t)+F^{\prime} \xi(t)-\int_{t}^{T} g(t) d t\right) \\
& -\int_{0}^{T} y(t)^{\prime} d\left(E^{\prime} \pi(t)-\int_{t}^{T} g(t) d t\right) \\
& =-\int_{0}^{T} y(t)^{\prime} d\left(E^{\prime} \pi(t)-\int_{t}^{T} g(t) d t\right)
\end{aligned}
$$

which implies that $\int_{0}^{T} y(t)^{\prime} d F^{\prime} \xi(t)$ exists. The existence of $\int_{0}^{T} \bar{z}(t)^{\prime} d \xi(t)$ now follows from the existence of both $\int_{0}^{T} y(t)^{\prime} d F^{\prime} \xi(t)$ and $\int_{0}^{T}(F y(t)+\bar{z}(t))^{\prime} d \xi(t)$ since

$$
\int_{0}^{T} \bar{z}(t)^{\prime} d \xi(t)=\int_{0}^{T}(F y(t)+\bar{z}(t))^{\prime} d \xi(t)-\int_{0}^{T} y(t)^{\prime} d F^{\prime} \xi(t)
$$

The requirement that $\pi(t)$ is bounded, measurable, and of finite variation in $\left(S C S C L P^{*}\right)$ is important, as it makes the integration by parts valid in the proof of the above proposition (see also Harrison [19]). As a consequence of the proof, we have the following corollary.

Corollary 1. Strong duality holds between (SCSCLP) and (SCSCLP $)$ if and only if there exist $(u(t), y(t))$ and $(\pi(t), \eta(t), \xi(t))$ which are feasible to $(S C S C L P)$ and $\left(S C S C L P^{*}\right)$, respectively, and satisfy the following conditions:

$$
\begin{array}{r}
\int_{0}^{T}\left(c(t)-G^{\prime} \pi(t)+H^{\prime} \eta(t)\right)^{\prime} u(t) d t=0 \\
\int_{0}^{T}(b(t)-H u(t))^{\prime} \eta(t) d t=0 \\
\int_{0}^{T}(h(t)-F y(t))^{\prime} d \xi(t)=0 .
\end{array}
$$

We call all three equations in (5) the complementary slackness condition for $(S C S C L P)$ and $\left(S C S C L P^{*}\right)$.

The following are standard definitions and notations which we will use throughout the remainder of the paper.

We call a sequence of time epochs $P=\left\{t_{0}, \ldots, t_{p}\right\}$ a partition of $[0, T]$ if

$$
0=t_{0} \leq t_{1} \leq \cdots \leq t_{p}=T
$$


We use $|P|$ to denote the cardinality of $P$. Note that since our development sometimes treats $t_{i}$ as a variable, we allow $t_{i}=t_{i-1}$ for some $i \geq 1$ and always treat $t_{i}$ and $t_{i-1}$ as two different variables.

We say that a function $f(t)$ is piecewise constant (linear) with a partition $P=$ $\left\{t_{0}, \ldots, t_{p}\right\}$ if $f(t)$ is constant (linear) on $\left[t_{i-1}, t_{i}\right)$ for $i=1, \ldots, p$. We say $f(t)$ is piecewise constant (linear) on $[0, T]$ if $f(t)$ is piecewise constant (linear) with some partition of $[0, T]$.

Let $P=\left\{t_{0}, \ldots, t_{p}\right\}$ be a partition of $[0, T]$. Throughout this paper, we assume Assumption 1 holds. In particular, we assume that $a(t), h(t)$, and $c(t)$ are piecewise linear and $b(t)$ and $g(t)$ are piecewise constant with partition $P$. Let $\mathcal{B}$ be the set of breakpoints of $a(t), b(t), c(t), g(t)$, and $h(t)$. For each breakpoint in $\mathcal{B}$, we select one element $t_{i}$ in $P$ such that its value denotes the same time in $[0, T]$ as the breakpoint. We always select $t_{0}=0$ and $t_{p}=T$. We denote $D^{P}$ to be the set of selected elements of $P$ excluding $t_{0}$ and $t_{p}$. Let $D_{1}^{P}=D^{P} \bigcup\left\{t_{0}, t_{p}\right\}$. We sometimes omit the superscript $P$ when the context is clear.

We say that an interval $\left[t_{i-1}, t_{i}\right]$ is a subinterval of $\left[t_{l}, t_{m}\right]$, where $t_{l}$ and $t_{m}$ are two consecutive breakpoints in $D_{1}^{P}$, if $l \leq i-1<i \leq m$. In this case, we also say that $t_{i-1}, t_{i}$, and $\left[t_{i-1}, t_{i}\right]$ reside on $\left[t_{l}, t_{m}\right]$.

For a function $f(t)$, we will use the notation

$$
f(t-)=\lim _{s \rightarrow t-} f(s) \quad \text { and } \quad f(t+)=\lim _{s \rightarrow t+} f(s),
$$

when the above limits exist and $t$ is not equal to any breakpoint in $D_{1}^{P}$. If $\left[t_{i-1}, t_{i}\right]$ is a zero-length subinterval of $\left[t_{l}, t_{m}\right]$, where $t_{l}$ and $t_{m}$ are two consecutive breakpoints in $D_{1}^{P}$, we let

$$
f\left(t_{i}-\right)= \begin{cases}\lim _{s \rightarrow t_{i}-} f(s) & \text { if } t_{i}=t_{m} \\ \lim _{s \rightarrow t_{i}+} f(s) & \text { if } t_{i}=t_{l}\end{cases}
$$

and let $f\left(t_{i-1}+\right)=f(t)=f\left(t_{i}-\right)$. We note that the value of $f\left(t_{i}\right)$ is sensitive to both the value of $t_{i}$ and its index $i$.

Given $t_{i} \neq t_{i-1}$ for $i=1, \ldots, p$ and a set of $2 p$ numbers $\hat{f}\left(t_{0}+\right), \hat{f}\left(t_{1}-\right), \hat{f}\left(t_{1}+\right), \ldots$, $\hat{f}\left(t_{p-1}+\right), \hat{f}\left(t_{p}-\right)$, the function $f(t)$ defined by

$$
f(t)= \begin{cases}\hat{f}\left(t_{i}+\right) & \text { if } t=t_{0}, t_{1}, \ldots, t_{p-1} \\ 0 & \text { if } t=T, \\ \frac{t_{i}-t}{t_{i}-t_{i-1}} \hat{f}\left(t_{i-1}+\right)+\frac{t-t_{i-1}}{t_{i}-t_{i-1}} \hat{f}\left(t_{i}-\right) & \text { for } t \in\left(t_{i-1}, t_{i}\right), i=1, \ldots, p\end{cases}
$$

is called the piecewise-linear extension of these $2 p$ numbers; for a set of $p$ numbers $\hat{f}\left(t_{0}+\right), \hat{f}\left(t_{1}+\right), \ldots, \hat{f}\left(t_{p-1}+\right)$, the function $f(t)$ defined by

$$
f(t)= \begin{cases}\hat{f}\left(t_{p-1}+\right) & t=T \\ \hat{f}\left(t_{i-1}+\right) & \text { for } t \in\left[t_{i-1}, t_{i}\right), i=1, \ldots, p\end{cases}
$$

is called the piecewise constant extension of these $p$ variables.

For two functions $f(t)$ and $g(t)$, we denote $\int_{a}^{b} f(t) d g(t)$ as the Lebesgue-Stieltjes integral of $f(t)$ with respect to $g(t)$ from $a$ to $b$, given that the integral exists, including both $a$ and $b$. For any mathematical program (LP) we let $V(\mathrm{LP})$ be the optimal value of the objective function, which may not be attained. For any feasible solution $x$ of (LP), we let $V(\mathrm{LP}, x)$ be the solution value of $x$ in (LP). For any $n$-dimensional 


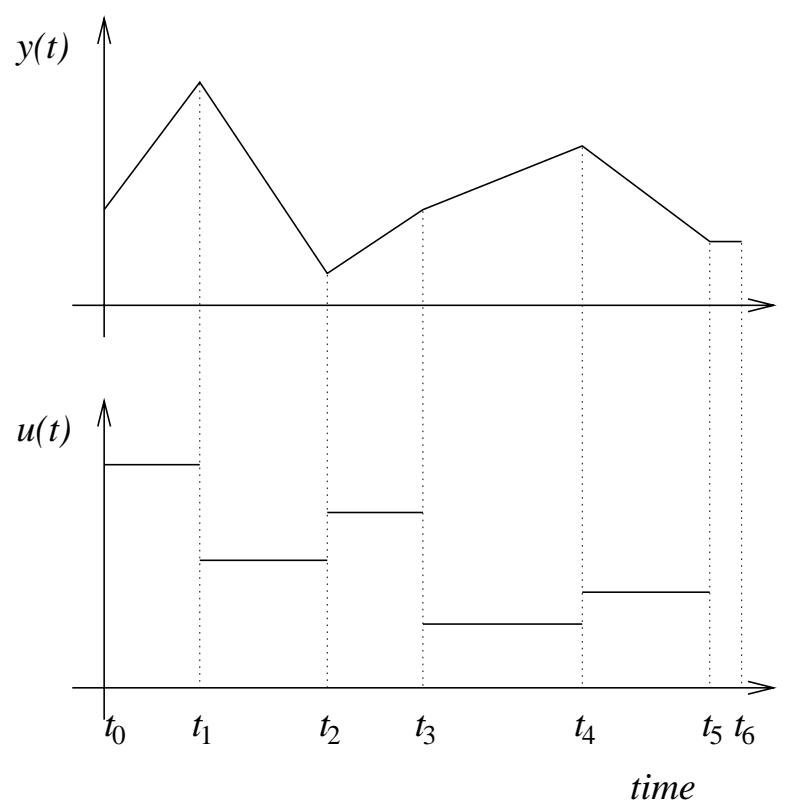

FIG. 1. A piecewise constant optimal control for (SCLP).

vector $x$, we denote by $x_{i}$ the $i$ th coordinate of $x$, and, for any nonempty subset $Q \subseteq\{1, \ldots, n\}$, we use $x_{Q},[x]_{Q}$, or $(x)_{Q}$ to denote the vector with components $x_{i}$, $i \in Q$ (with $x_{i}$ arranged in the same order as in $x$ ). For a matrix $A$, we denote by $A_{i j}$ the $j$ th element of the $i$ th row of matrix $A$ and denote by $A_{i \bullet}$ the $i$ th row of $A$.

3. A quadratic programming subproblem. By a result of Pullan [42] (see also Anderson and Nash [2]), there exists an optimal basic feasible solution to (SCLP) whose $u(t)$ is piecewise constant (see Figure 1) when Assumption 1 holds and the solution set to $(S C L P)$ is bounded. We will prove later in the paper that this remains true for $(S C S C L P)$. For any feasible control $u(t)$ that is piecewise constant with respect to a partition $P$, we have the following standard linear approximation problem (see Pullan [41] and the references therein):

$$
\begin{aligned}
D P(P) \min & \sum_{i=1}^{p}\left(t_{i}-t_{i-1}\right) \hat{u}\left(t_{i-1}+\right)^{\prime} c\left(\frac{t_{i}+t_{i-1}}{2}\right) \\
& +\sum_{i=1}^{p} \frac{t_{i}-t_{i-1}}{2}\left(\hat{y}\left(t_{i}\right)+\hat{y}\left(t_{i-1}\right)\right)^{\prime} g\left(t_{i-1}+\right) \\
\text { s.t. } & E \hat{y}\left(t_{0}\right)=a\left(t_{0}\right), \\
& \left(t_{i}-t_{i-1}\right) G \hat{u}\left(t_{i-1}+\right)+E \hat{y}\left(t_{i}\right)-E \hat{y}\left(t_{i-1}\right)=a\left(t_{i}\right)-a\left(t_{i-1}\right), \\
& i=1, \ldots, p, \\
& H \hat{u}\left(t_{i-1}+\right) \leq b\left(t_{i-1}+\right), \quad i=1, \ldots, p, \\
& F \hat{y}\left(t_{i}\right) \leq h\left(t_{i}\right), \quad i=0, \ldots, p, \\
& \hat{u}\left(t_{i-1}+\right) \geq 0, \quad i=1, \ldots, p,
\end{aligned}
$$


where we have the convention that $c\left(\frac{t_{i}+t_{i-1}}{2}\right)=c\left(t_{i}-\right)$ whenever $t_{i}=t_{i-1}$. Note that even though it is possible that $t_{i}=t_{i-1}$ for some $i \geq 1$, we still treat $\hat{u}\left(t_{i}+\right)$ and $\hat{u}\left(t_{i-1}+\right)$ as separate variables. If $(\hat{u}, \hat{y})$ is a feasible solution to $D P(P)$, where partition $P$ satisfies $t_{i} \neq t_{i-1}$ for all $i$, the piecewise constant extension of $\hat{u}$, together with the piecewise-linear extension of $\hat{y}$, defines a feasible solution to $(S C S C L P)$ with the same cost, due to Assumption 1. If we fix the partition, $D P(P)$ is a linear programming problem. So, once an optimal partition $P$ is known, an optimal solution can be computed by solving the linear program $D P(P)$.

However, we do not know the optimal partition in advance. The algorithms proposed by Pullan [41] and by Philpott and Craddock [39] alternatively do the following two steps:

1) Improve the control for the current partition.

2) Improve the partition.

In contrast, the algorithm we propose improves both the control and partition at the same time.

By introducing new variables

$$
\hat{v}\left(t_{i}\right)=\left(t_{i}-t_{i-1}\right) \hat{u}\left(t_{i-1}+\right),
$$

we can eliminate variable $\hat{u}$ from $D P(P)$ and obtain the following simpler mathematical programming problem in variables $\hat{v}, \hat{y}$, and $\hat{t}$, with $\hat{t}$ being the vector of $t_{i}$ 's such that $t_{i} \notin D_{1}^{P}$ :

$$
\begin{aligned}
Q P(|P|) \min & \sum_{i=1}^{p} \hat{v}\left(t_{i}\right)^{\prime} c\left(\frac{t_{i}+t_{i-1}}{2}\right)+\sum_{i=1}^{p} \frac{t_{i}-t_{i-1}}{2}\left(\hat{y}\left(t_{i}\right)+\hat{y}\left(t_{i-1}\right)\right)^{\prime} g\left(t_{i-1}+\right) \\
\text { s.t. } & E \hat{y}\left(t_{0}\right)=a\left(t_{0}\right) \\
& \quad G \hat{v}\left(t_{i}\right)+E \hat{y}\left(t_{i}\right)-E \hat{y}\left(t_{i-1}\right)=a\left(t_{i}\right)-a\left(t_{i-1}\right) \\
& \quad i=1, \ldots, p \\
& H \hat{v}\left(t_{i}\right) \leq\left(t_{i}-t_{i-1}\right) b\left(t_{i-1}+\right), \quad i=1, \ldots, p \\
& F \hat{y}\left(t_{i}\right) \leq h\left(t_{i}\right), \quad i=0, \ldots, p \\
& 0=t_{0} \leq t_{1} \leq \ldots \leq t_{p}=T \\
& \hat{v}\left(t_{i}\right) \geq 0, \quad i=1, \ldots, p
\end{aligned}
$$

where $c\left(\frac{t_{i}+t_{i-1}}{2}\right)=c\left(t_{i}-\right)$ whenever $t_{i}=t_{i-1}$. Note that the breakpoints in $D_{1}^{P}$ are fixed and are not variables. We treat both $\hat{v}\left(t_{i}\right)$ and $\hat{y}\left(t_{i}\right)$ as variables. Let $t_{l}$ and $t_{m}$ be two consecutive breakpoints in $D_{1}^{P}$. For any $i \in(l, m], c\left(\frac{t_{i}+t_{i-1}}{2}\right), a\left(t_{i}\right)-a\left(t_{i-1}\right)$, and $h\left(t_{i}\right)$ are the following linear functions of $t_{i}$ and $t_{i-1}$ (note that by Assumption 1b, $c(t), a(t)$, and $h(t)$ are piecewise linear and therefore $\dot{c}(t), \dot{a}(t)$, and $\dot{h}(t)$ are piecewise constant):

$$
\begin{aligned}
c\left(\frac{t_{i}+t_{i-1}}{2}\right) & =c\left(t_{l}\right)+\frac{t_{i}+t_{i-1}-2 t_{l}}{2} \dot{c}\left(t_{l}+\right), \\
a\left(t_{i}\right)-a\left(t_{i-1}\right) & =\left(t_{i}-t_{i-1}\right) \dot{a}\left(t_{l}+\right), \\
h\left(t_{i}\right) & =h\left(t_{l}\right)+\left(t_{i}-t_{l}\right) \dot{h}\left(t_{l}+\right),
\end{aligned}
$$

and $g\left(t_{i-1}+\right)=g\left(t_{l}\right)$ and $b\left(t_{i-1}+\right)=b\left(t_{l}\right)$ are constant vectors. So, $Q P(|P|)$ is a quadratic programming problem with polyhedral constraints.

Given a feasible solution $(\hat{v}, \hat{y}, \hat{t})$ to $Q P(|P|)$ such that $t_{i} \neq t_{i-1}$ for all $i$, we can obtain a feasible solution $(\hat{u}, \hat{y})$ to problem $D P(P)$ with $P$ defined from vector $\hat{t}$ and 
the breakpoints in $D_{1}^{P}$ and $\hat{u}$ defined from

$$
\hat{u}\left(t_{i-1}+\right)=\frac{\hat{v}\left(t_{i}\right)}{t_{i}-t_{i-1}} .
$$

Equation (6) defines an injective mapping from the solution set to $D P(P)$ to the solution set to $Q P(|P|)$. The two related solutions have the same solution value.

However, if $t_{i}=t_{i-1}$ but $\hat{v}\left(t_{i}\right) \neq 0$ for some $i$, the right-hand side of (8) is not properly defined, i.e., there may be a solution to $Q P(|P|)$ for which the corresponding solution to $D P(P)$ cannot be constructed. We overcome this difficulty by constantly removing redundant zero-length intervals in a feasible solution and by using only the solution $(\hat{v}, \hat{y}, \hat{t})$ to $Q P(|P|)$ that satisfies

$$
t_{i} \neq t_{i-1} \quad \text { for all } \quad i \geq 1
$$

to construct a feasible solution for $D P(P)$ (and so for $(S C S C L P)$ ). When some zerolength intervals cannot be removed, we show that there is a series of feasible solutions to $Q P(|P|)$ that satisfies (9) whose solution value becomes arbitrarily close to that of the feasible solution to $Q P(|P|)$. This is key to understanding the absence of a duality gap result between $(S C S C L P)$ and $\left(S C S C L P^{*}\right)$, as we will see later on.

Lemma 1. Suppose $u(t)$ in all feasible solutions to $(S C S C L P)$ is bounded. Let $(\hat{v}, \hat{y}, \hat{t})$ be a feasible solution to $Q P(|P|)$. Then

$$
\hat{v}\left(t_{i}\right)=0 \quad \text { whenever } \quad t_{i}=t_{i-1} .
$$

Proof. Suppose $t_{i}=t_{i-1}$ for some $i$, but $\hat{v}\left(t_{i}\right) \neq 0$. Let $\left[t_{l}, t_{m}\right]$ be the interval $t_{i}$ resides on, where $t_{l}$ and $t_{m}$ are two consecutive breakpoints in $D_{1}^{P}$. Without loss of generality, we may assume that there exists a positive-length subinterval of $\left[t_{l}, t_{m}\right]$ that is adjacent to $\left[t_{i-1}, t_{i}\right]$ (since we can switch the values of $\hat{v}\left(t_{j}\right)$ and $\hat{y}\left(t_{j}\right)$ with adjacent zero-length subintervals on $\left[t_{l}, t_{m}\right]$ and maintain the feasibility of the solution). We assume that the adjacent positive-length subinterval on $\left[t_{l}, t_{m}\right]$ is $\left[t_{i-2}, t_{i-1}\right]$. When the adjacent positive-length subinterval of $\left[t_{l}, t_{m}\right]$ is $\left[t_{i}, t_{i+1}\right]$, a similar analysis applies.

For any $\tau \in(0,1)$, it is easy to verify that the following solution is feasible for $Q P(|P|)$ :

$$
\begin{aligned}
& \tilde{t}_{j}^{\tau}= \begin{cases}t_{j} & \text { if } j \neq i-1 \text { and } j \neq i, \\
\tau t_{i-2}+(1-\tau) t_{i-1} & \text { if } j=i-1, \\
t_{i} & \text { if } j=i,\end{cases} \\
& \tilde{v}^{\tau}\left(t_{j}^{\tau}\right)= \begin{cases}\hat{v}\left(t_{j}\right) & \text { if } j \neq i-1 \text { and } j \neq i, \\
(1-\tau) \hat{v}\left(t_{i-1}\right) & \text { if } j=i-1, \\
\tau \hat{v}\left(t_{i-1}\right)+\hat{v}\left(t_{i}\right) & \text { if } j=i,\end{cases} \\
& \tilde{y}^{\tau}\left(t_{j}^{\tau}\right)= \begin{cases}\hat{y}\left(t_{j}\right) & \text { if } j \neq i-1 \text { and } j \neq i, \\
\tau \hat{y}\left(t_{i-2}\right)+(1-\tau) \hat{y}\left(t_{i-1}\right) & \text { if } j=i-1, \\
\hat{y}\left(t_{i}\right) & \text { if } j=i .\end{cases}
\end{aligned}
$$

The basic idea is to split interval $\left[t_{i-2}, t_{i-1}\right]$ into two intervals $\left[t_{i-2}, \tau t_{i-2}+(1-\tau) t_{i-1}\right]$ and $\left[\tau t_{i-2}+(1-\tau) t_{i-1}, t_{i-1}\right]$ and combine the second interval with $\left[t_{i-1}, t_{i}\right]$. It is easy to check that $\left(\tilde{v}^{\tau}, \tilde{y}^{\tau}, \tilde{t}^{\tau}\right)$ is feasible for $Q P(|P|)$ and has one less zero-length interval than $(\hat{v}, \hat{y}, \hat{t})$. Applying the same process repeatedly, we can eliminate all the zero-length intervals in the solution $(\hat{v}, \hat{y}, \hat{t})$. 
Let $\left(\bar{v}^{\tau}, \bar{y}^{\tau}, \bar{t}^{\tau}\right)$ be the resulting solution and let $Q$ be the resulting partition. Hence, $\left(\bar{v}^{\tau}, \bar{y}^{\tau}, \bar{t}^{\tau}\right)$ is feasible for $Q P(|Q|)$. From this solution, we can construct a feasible solution for $D P(Q)$ (and thus for $(S C S C L P)$ ) by using (8). However, as $\tau$ tends to zero, the corresponding feasible solution to $D P(P)$ is unbounded from above (since the denominator in (8) goes to zero, but the numerator is bounded away from zero). Thus $u(t)$ in $(S C S C L P)$ is unbounded and this creates a contradiction.

We remark that Lemma 1 implies that if $u(t)$ is bounded and $E$ is an identity matrix (e.g., a bounded and feasible $(S C L P)$ ), then $\hat{y}\left(t_{i-1}\right)=\hat{y}\left(t_{i}\right)$ whenever $t_{i-1}=$ $t_{i}$. In general, when (10) holds, it is possible that $\hat{y}\left(t_{i-1}\right) \neq \hat{y}\left(t_{i}\right)$ even if $t_{i-1}=t_{i}$. If in addition to (10), $\hat{y}\left(t_{i-1}\right)=\hat{y}\left(t_{i}\right)$ for some $i$ such that $t_{i-1}=t_{i}$, then we can eliminate the zero-length interval $\left[t_{i-1}, t_{i}\right]$ from $(\hat{v}, \hat{y}, \hat{t})$ while maintaining the feasibility and improving the solution value of the solution. This fact will be used later in section 4 to remove redundant intervals.

In general, $u(t)$ may not be bounded in a feasible solution to $(S C S C L P)$. It is possible that there is no feasible solution to $(S C S C L P)$ that is optimal for $(S C S C L P)$. This perhaps is the key difficulty in establishing the absence of a duality gap between $(S C S C L P)$ and $\left(S C S C L P^{*}\right)$ by conventional methods. Hence, we have the following relationship between $(S C S C L P)$ and $Q P(|P|)$.

LEMma 2. Given any feasible solution $(\hat{v}, \hat{y}, \hat{t})$ to $Q P(|P|)$, there exists a series of feasible solutions $\left(\hat{v}^{k}, \hat{y}^{k}, \hat{t}^{k}\right)$ to $Q P(|P|)$ that satisfies (9) and whose solution value becomes arbitrarily close to that of $(\hat{v}, \hat{y}, \hat{t})$ as $k$ tends to infinity.

Proof. By using the same procedure used to prove Lemma 1, we can construct a solution $\left(\bar{v}^{\tau}, \bar{y}^{\tau}, \bar{t}^{\tau}\right)$ which is feasible to $Q P(|Q|)$ for some partition $Q$ and satisfies (9). It is easily verified that the solution value of $\left(\bar{v}^{\tau}, \bar{y}^{\tau}, \bar{t}^{\tau}\right)$ to $Q P(|Q|)$ becomes arbitrarily close to that of $(\hat{v}, \hat{y}, \hat{t})$ as $\tau$ goes to zero.

In fact, we can have $t_{i} \neq t_{i+1}$ and $t_{i-1} \neq t_{i-2}$ whenever $t_{i}=t_{i-1}$ in a local optimum for $Q P(|P|)$. The existence of $\hat{v}\left(t_{i}\right) \neq 0$ but $t_{i}=t_{i-1}$ indicates the presence of the Dirac $\delta$ function in $u(t)$ at time $t_{i}$.

A direct consequence of Lemma 2 is $V((S C S C L P)) \leq V(Q P(|P|))$ for all $P$. This fact enables us to solve $(S C S C L P)$ through solving $Q P(|P|)$ for a series of partitions. We note that $V(Q P(|P|))=V(S C S C L P)$ does not imply that there is a feasible solution for $(S C S C L P)$, whose solution value is equal to $V(Q P|P|)$, due to the possible presence of zero-length intervals in $P$.

3.1. Finding a KKT point for $\boldsymbol{Q P}(|\boldsymbol{P}|)$. We do not need to solve the nonconvex quadratic program $Q P(|P|)$ to optimality, as we will see in section 6 . We only need to compute a series of KKT points (or equivalently, stationary points) of a set of quadratic programs. We use the Frank-Wolfe method (see Martos [33] and Murty [36]) or general matrix-splitting algorithms (see Lin and Pang [28], Eckstein [11], Bertsekas and Tsitsiklis [9], Luo and Tseng [31]) to find a KKT point for the quadratic program. There are other methods for obtaining a KKT point, such as those proposed by Ye [51] and Kojima, Noma, and Yoshise [24].

4. Removing redundant intervals. After finding a KKT point of $Q P(|P|)$, it is possible that some zero-length intervals can be removed, as we noted following Lemma 1. It is also possible that some adjacent intervals can be merged while improving the solution value. The reduction of unnecessary control pieces in the solution is a key feature of the new algorithm. This enables us to prove the convergence of the new algorithm without requiring the norm of the maximal length interval in the discretization to tend to zero (cf. Pullan [41] and Philpott and Craddock [39]). 
To do this, let $(\hat{v}, \hat{y}, \hat{t})$ be a feasible solution to $Q P(|P|)$ and let $\left[t_{i-1}, t_{i}\right]$ and $\left[t_{i}, t_{i+1}\right]$ be two adjacent intervals that reside on $\left[t_{l}, t_{m}\right]$, where $t_{l}$ and $t_{m}$ are two consecutive breakpoints in $D_{1}^{P}$. We eliminate $t_{i}$ from $P$ (or equivalently, combine $\left[t_{i-1}, t_{i}\right]$ and $\left.\left[t_{i}, t_{i+1}\right]\right)$ and define a new feasible solution $(\tilde{v}, \tilde{y}, \tilde{t})$ for $Q P\left(\left|P \backslash\left\{t_{i}\right\}\right|\right)$ as follows. Let $\tilde{v}$ be the vector formed by removing $\hat{v}\left(t_{i+1}\right)$ from $\hat{v}$ and then replacing $\hat{v}\left(t_{i}\right)$ with $\hat{v}\left(t_{i}\right)+\hat{v}\left(t_{i+1}\right)$, let $\tilde{y}$ be the vector formed by removing $\hat{y}\left(t_{i}\right)$ from $\hat{y}$, and let $\tilde{t}$ be the vector formed by removing $t_{i}$ from $\hat{t}$.

Lemma 3. Let $\left[t_{i-1}, t_{i}\right]$ and $\left[t_{i}, t_{i+1}\right]$ be two adjacent intervals that reside on $\left[t_{l}, t_{m}\right]$, where $t_{l}$ and $t_{m}$ are two consecutive breakpoints in $D_{1}^{P}$. If

$$
\left(t_{i+1}-t_{i}\right) \hat{v}\left(t_{i}\right)^{\prime} \dot{c}\left(t_{i-1}+\right)+\left(t_{i+1}-t_{i}\right) \hat{y}\left(t_{i-1}\right)^{\prime} g\left(t_{i-1}+\right)+\left(t_{i}-t_{i-1}\right) \hat{y}\left(t_{i+1}\right)^{\prime} g\left(t_{i-1}+\right)
$$

$$
\leq\left(t_{i}-t_{i-1}\right) \hat{v}\left(t_{i+1}\right)^{\prime} \dot{c}\left(t_{i-1}+\right)+\left(t_{i+1}-t_{i-1}\right) \hat{y}\left(t_{i}\right)^{\prime} g\left(t_{i-1}+\right)
$$

then we can combine $\left[t_{i-1}, t_{i}\right]$ and $\left[t_{i}, t_{i+1}\right]$ while maintaining the feasibility and improving the solution value of a feasible solution to $Q P(|P|)$.

Proof. The difference between the solution value of $(\hat{v}, \hat{y}, \hat{t})$ and that of the solution $(\tilde{v}, \tilde{y}, \tilde{t})$ is the following:

$$
\begin{aligned}
& \hat{v}\left(t_{i}\right)^{\prime} c\left(\frac{t_{i}+t_{i-1}}{2}\right)+\hat{v}\left(t_{i+1}\right)^{\prime} c\left(\frac{t_{i+1}+t_{i}}{2}\right)+\frac{t_{i}-t_{i-1}}{2}\left(\hat{y}\left(t_{i}\right)+\hat{y}\left(t_{i-1}\right)\right)^{\prime} g\left(t_{i-1}+\right) \\
& +\frac{t_{i+1}-t_{i}}{2}\left(\hat{y}\left(t_{i+1}\right)+\hat{y}\left(t_{i}\right)\right)^{\prime} g\left(t_{i-1}+\right)-\left(\hat{v}\left(t_{i}\right)+\hat{v}\left(t_{i+1}\right)\right)^{\prime} c\left(\frac{t_{i+1}+t_{i-1}}{2}\right) \\
& -\frac{t_{i+1}-t_{i-1}}{2}\left(\hat{y}\left(t_{i+1}\right)+\hat{y}\left(t_{i-1}\right)\right)^{\prime} g\left(t_{i-1}+\right) \\
= & -\frac{1}{2}\left(\left(t_{i+1}-t_{i}\right) \hat{v}\left(t_{i}\right)^{\prime} \dot{c}\left(t_{i-1}+\right)+\left(t_{i+1}-t_{i}\right) \hat{y}\left(t_{i-1}\right)^{\prime} g\left(t_{i-1}+\right)\right. \\
& \left.+\left(t_{i}-t_{i-1}\right) \hat{y}\left(t_{i+1}\right)^{\prime} g\left(t_{i-1}+\right)\right) \\
& +\frac{1}{2}\left(\left(t_{i}-t_{i-1}\right) \hat{v}\left(t_{i+1}\right)^{\prime} \dot{c}\left(t_{i-1}+\right)+\left(t_{i+1}-t_{i-1}\right) \hat{y}\left(t_{i}\right)^{\prime} g\left(t_{i-1}+\right)\right) .
\end{aligned}
$$

We see that the new solution has a smaller solution value if and only if (11) holds.

A direct corollary to Lemma 3 is the following.

Corollary 2. Let $t_{l}$ and $t_{m}$ be two consecutive breakpoints in $D_{1}^{P}$. We can combine adjacent zero-length intervals in $\left[t_{l}, t_{m}\right]$ while maintaining the feasibility and improving the solution value of a feasible solution to $Q P(|P|)$.

Proof. Let $\left[t_{i-1}, t_{i}\right]$ and $\left[t_{i}, t_{i+1}\right]$ be two adjacent zero-length intervals that reside on $\left[t_{l}, t_{m}\right]$. Since $t_{i-1}=t_{i}=t_{i+1},(11)$ is trivially satisfied. By Lemma 3 , we can combine $\left[t_{i-1}, t_{i}\right]$ and $\left[t_{i}, t_{i+1}\right]$ and maintain the feasibility and improve the solution value of the feasible solution to $Q P(|P|)$.

By Corollary 2, we can combine adjacent zero-length intervals. The following lemma implies that all the zero-length intervals except those at the breakpoints in $D_{1}^{P}$ can be eliminated.

Lemma 4. Let $\left[t_{i-1}, t_{i}\right]$ be a zero-length interval that resides on $\left[t_{l}, t_{m}\right]$, where $t_{l}$ and $t_{m}$ are two consecutive breakpoints in $D_{1}^{P}$. Suppose $\left[t_{i-2}, t_{i-1}\right]$ and $\left[t_{i}, t_{i+1}\right]$ are two positive-length intervals that also reside on $\left[t_{l}, t_{m}\right]$. We can either

(a) combine $\left[t_{i-2}, t_{i-1}\right]$ and $\left[t_{i-1}, t_{i}\right]$, or

(b) combine $\left[t_{i-1}, t_{i}\right]$ and $\left[t_{i}, t_{i+1}\right]$, 
while maintaining the feasibility and improving the solution value of the feasible solution to $Q P(|P|)$.

Proof. Since $t_{i-1}=t_{i}$, by Lemma 3 , we can combine $\left[t_{i-2}, t_{i-1}\right]$ and $\left[t_{i-1}, t_{i}\right]$ if the following relation holds:

$$
\left(t_{i-1}-t_{i-2}\right) \hat{y}\left(t_{i}\right)^{\prime} g\left(t_{i-2}+\right) \leq\left(t_{i-1}-t_{i-2}\right)\left(\hat{v}\left(t_{i}\right)^{\prime} \dot{c}\left(t_{i-2}+\right)+\hat{y}\left(t_{i-1}\right)^{\prime} g\left(t_{i-2}+\right)\right) .
$$

By Lemma 3 again, we can combine $\left[t_{i-1}, t_{i}\right]$ and $\left[t_{i}, t_{i+1}\right]$ if the following relation holds:

$$
\left(t_{i+1}-t_{i}\right) \hat{y}\left(t_{i}\right)^{\prime} g\left(t_{i}+\right) \geq\left(t_{i+1}-t_{i}\right)\left(\hat{v}\left(t_{i}\right)^{\prime} \dot{c}\left(t_{i}+\right)+\hat{y}\left(t_{i-1}\right)^{\prime} g\left(t_{i}+\right)\right) .
$$

By assumption, we have $t_{i+1}-t_{i}>0$ and $t_{i-1}-t_{i-2}>0$. Since $c(t)$ is linear and $g(t)$ is constant on $\left[t_{l}, t_{m}\right]$, we have

$$
g\left(t_{i-2}+\right)=g\left(t_{i}+\right) \quad \text { and } \quad \dot{c}\left(t_{i-2}+\right)=\dot{c}\left(t_{i}+\right)
$$

So either (12) or (13) is true. This proves the lemma.

We next propose the following procedure for removing redundant intervals on $\left[t_{l}, t_{m}\right]$, where $t_{l}$ and $t_{m}$ are two consecutive breakpoints in $D_{1}^{P}$.

Procedure PURIFY. Repeatedly combine two adjacent intervals $\left[t_{i-1}, t_{i}\right]$ and $\left[t_{i}, t_{i+1}\right]$ in $\left[t_{l}, t_{m}\right]$ if $(11)$ is satisfied.

When more than one pair of adjacent intervals satisfies (11), we can combine them in an arbitrary order, one pair at a time. Let $\tilde{P}$ be the resulting partition of $[0, T]$ after we apply the above procedure to $P$ for all consecutive breakpoints in $D_{1}^{P}$. We call $\tilde{P}$ a purified partition of $[0, T]$. Note that the remaining zero-length intervals are located at the breakpoints in $D_{1}^{P}$ and there are at most $2\left|D_{1}^{P}\right|$ zero-length intervals in $P$.

5. Improving a nonoptimal solution. One major step of the new algorithm is to calculate a KKT point of the system $Q P(|P|)$ for some partition $P$ of $[0, T]$. However, the problem $Q P(|P|)$ is nonconvex. To obtain a global optimal solution for ( $S C S C L P$ ), we must be able to improve a solution that is not globally optimal for $(S C S C L P)$. In this section, we give descent directions for solutions that are not globally optimal for $(S C S C L P)$. To do so, we first introduce a new discrete approximation for $(S C S C L P)$ which is closely related to the dual problem $\left(S C S C L P^{*}\right)$. From this new approximation, we derive a criterion that detects whether a solution is globally optimal for $(S C S C L P)$. If this criterion is not satisfied, we give a descent direction for the current solution and thus improve the solution value. We show that instead of using the direction constructed in section 5.3, an algorithm for (SCSCLP) can also use the Frank-Wolfe method or the matrix-splitting algorithm to find a descent direction. We also show that the first iterate of the Frank-Wolfe method provides an upper bound on the current duality gap.

5.1. A new discrete approximation. For partition $P=\left\{t_{0}, \ldots, t_{p}\right\}$, we let $P^{\prime}=\left\{t_{0}, \frac{t_{0}+t_{1}}{2}, t_{1}, \ldots, \frac{t_{p-1}+t_{p}}{2}, t_{p}\right\}$ be a refined partition of $P$. Consider the following new discrete approximation to $(S C S C L P)$, a close variation of the second discretization in Pullan [41]:

$$
A P 1(P) \quad \min \sum_{i=1}^{p} \frac{t_{i}-t_{i-1}}{2}\left(c\left(t_{i-1}+\right)^{\prime} \hat{u}\left(t_{i-1}+\right)+c\left(t_{i}-\right)^{\prime} \hat{u}\left(t_{i}-\right)\right.
$$




$$
\left.+2 \hat{y}\left(\frac{t_{i-1}+t_{i}}{2}\right)^{\prime} g\left(t_{i-1}+\right)\right)
$$

s.t. $E \hat{y}\left(t_{0}\right)=a\left(t_{0}\right)$,

$$
\begin{array}{ll}
\left(\frac{t_{i}-t_{i-1}}{2}\right) G \hat{u}\left(t_{i}-\right)+E \hat{y}\left(t_{i}\right)-E \hat{y}\left(\frac{t_{i}+t_{i-1}}{2}\right) & \\
=a\left(t_{i}\right)-a\left(\frac{t_{i}+t_{i-1}}{2}\right), & i=1, \ldots, p, \\
\left(\frac{t_{i}-t_{i-1}}{2}\right) G \hat{u}\left(t_{i-1}+\right)+E \hat{y}\left(\frac{t_{i}+t_{i-1}}{2}\right)-E \hat{y}\left(t_{i-1}\right) & \\
=a\left(\frac{t_{i}+t_{i-1}}{2}\right)-a\left(t_{i-1}\right), & i=1, \ldots, p, \\
H \hat{u}\left(t_{i-1}+\right) \leq b\left(t_{i-1}+\right), & i=1, \ldots, p, \\
H \hat{u}\left(t_{i}-\right) \leq b\left(t_{i}-\right), & i=1, \ldots, p, \\
F \hat{y}\left(t_{i}\right) \leq h\left(t_{i}\right), & i=1, \ldots, p, \\
F \hat{y}\left(\frac{t_{i}+t_{i-1}}{2}\right) \leq h\left(\frac{t_{i}+t_{i-1}}{2}\right), & i=1, \ldots, p .
\end{array}
$$

Problem $A P 1(P)$ is closely related to the dual problem. The linear programming dual of $A P 1(P)$ gives rise to feasible solutions for the dual problem $\left(S C S C L P^{*}\right)$. Thus an optimal solution to $A P 1(P)$ contains the dual information. We will construct a descent solution for $(S C S C L P)$ based on a solution for $A P(P)$, a closely related linear program, to be defined shortly.

It is clear that the set of feasible solutions to $A P 1(P)$ is the same as the set of feasible solutions to $D P\left(P^{\prime}\right)$ if we identify $\hat{u}\left(t_{i}-\right)$ in $A P 1(P)$ with $\hat{u}\left(\left(\frac{t_{i-1}+t_{i}}{2}\right)+\right)$ in $D P\left(P^{\prime}\right)$. There are two differences between $D P\left(P^{\prime}\right)$ and $A P 1(P)$, both of which reside in the objective function. First, instead of averaging the cost coefficients of $u(t)$ over each subinterval, the instantaneous values of the cost coefficients at the original breakpoints of $P$ are used. Second, instead of using the average values of the state variable $y(t)$ in each subinterval, the values of $y(t)$ at the midpoint of each subinterval of $P$ are used. It can be checked that any feasible solution for $D P(P)$ defines a feasible solution for $D P\left(P^{\prime}\right)$ and thus for $A P 1(P)$, and these two solutions have the same solution value.

Similar to $Q P(|P|)$, we introduce $\hat{v}$ to eliminate $\hat{u}$, where

$$
\hat{v}\left(t_{i-1}+\right)=\frac{t_{i}-t_{i-1}}{2} \hat{u}\left(t_{i-1}+\right) \quad \text { and } \quad \hat{v}\left(t_{i}-\right)=\frac{t_{i}-t_{i-1}}{2} \hat{u}\left(t_{i}-\right) .
$$

Now $A P 1(P)$ is transformed into the following linear program in $\hat{v}$ and $\hat{y}$ :

$$
A P(P) \quad \min \sum_{i=1}^{p}\left(c\left(t_{i-1}+\right)^{\prime} \hat{v}\left(t_{i-1}+\right)+c\left(t_{i}-\right)^{\prime} \hat{v}\left(t_{i}-\right)\right.
$$




$$
\begin{aligned}
& \left.+\left(t_{i}-t_{i-1}\right) \hat{y}\left(\frac{t_{i-1}+t_{i}}{2}\right)^{\prime} g\left(t_{i-1}+\right)\right) \\
& \text { s.t. } E \hat{y}\left(t_{0}\right)=a\left(t_{0}\right) \text {, } \\
& G \hat{v}\left(t_{i}-\right)+E \hat{y}\left(t_{i}\right)-E \hat{y}\left(\frac{t_{i}+t_{i-1}}{2}\right)=a\left(t_{i}\right)-a\left(\frac{t_{i}+t_{i-1}}{2}\right), \\
& i=1, \ldots, p, \\
& G \hat{v}\left(t_{i-1}+\right)+E \hat{y}\left(\frac{t_{i}+t_{i-1}}{2}\right)-E \hat{y}\left(t_{i-1}\right)=a\left(\frac{t_{i}+t_{i-1}}{2}\right)-a\left(t_{i-1}\right), \\
& i=1, \ldots, p, \\
& H \hat{v}\left(t_{i-1}+\right) \leq\left(\frac{t_{i}-t_{i-1}}{2}\right) b\left(t_{i-1}+\right), \quad i=1, \ldots, p, \\
& H \hat{v}\left(t_{i}-\right) \leq\left(\frac{t_{i}-t_{i-1}}{2}\right) b\left(t_{i}-\right), \quad i=1, \ldots, p, \\
& F \hat{y}\left(t_{i}\right) \leq h\left(t_{i}\right), \quad i=0, \ldots, p, \\
& F \hat{y}\left(\frac{t_{i}+t_{i-1}}{2}\right) \leq h\left(\frac{t_{i}+t_{i-1}}{2}\right), \quad i=1, \ldots, p, \\
& \hat{v}\left(t_{i}-\right), \hat{v}\left(t_{i-1}+\right) \geq 0, \quad i=1, \ldots, p \text {. }
\end{aligned}
$$

Similar to $A P 1(P)$ and $D P\left(P^{\prime}\right), A P(P)$ and $Q P\left(\left|P^{\prime}\right|\right)$ have the same feasible solution set if the partition in $Q P\left(\left|P^{\prime}\right|\right)$ is fixed to $P^{\prime}$. We note that the actual value of $\hat{y}\left(t_{0}\right)$ does not affect the objective value of $A P(P)$ as long as $E \hat{y}\left(t_{0}\right)=a\left(t_{0}\right)$ and $F \hat{y}\left(t_{0}\right) \leq h\left(t_{0}\right)$ (which is indeed feasible by assumption). The dual problem for $A P(P)$ (after eliminating $\left.\hat{y}\left(t_{0}\right)\right)$ can be written as

$$
\begin{aligned}
A P^{*}(P) \max & \hat{\pi}\left(t_{0}+\right)^{\prime} a\left(t_{0}\right) \\
& +\sum_{i=1}^{p}\left(\hat{\pi}\left(t_{i-1}+\right)+\hat{\pi}\left(t_{i}-\right)\right)^{\prime}\left(a\left(t_{i}\right)-a\left(\frac{t_{i}+t_{i-1}}{2}\right)\right) \\
& -\sum_{i=1}^{p}\left(\frac{t_{i}-t_{i-1}}{2}\right)\left(\hat{\eta}\left(t_{i-1}+\right)+\hat{\eta}\left(t_{i}-\right)\right)^{\prime} b\left(t_{i}-\right) \\
& +\sum_{i=1}^{p}\left(\hat{\xi}\left(t_{i}\right)^{\prime} h\left(t_{i}\right)+\hat{\xi}\left(\frac{t_{i-1}+t_{i}}{2}\right)^{\prime} h\left(\frac{t_{i-1}+t_{i}}{2}\right)\right) \\
\text { s.t. } & c\left(t_{i}-\right)-G^{\prime} \hat{\pi}\left(t_{i}-\right)+H^{\prime} \hat{\eta}\left(t_{i}-\right) \geq 0, \quad i=1, \ldots, p, \\
& c\left(t_{i-1}+\right)-G^{\prime} \hat{\pi}\left(t_{i-1}+\right)+H^{\prime} \hat{\eta}\left(t_{i-1}+\right) \geq 0, i=1, \ldots, p, \\
& E^{\prime}\left(-\hat{\pi}\left(t_{i}-\right)+\hat{\pi}\left(t_{i-1}+\right)\right)+F^{\prime} \hat{\xi}\left(\frac{t_{i-1}+t_{i}}{2}\right)=\left(t_{i}-t_{i-1}\right) g\left(t_{i-1}+\right), \\
& E^{\prime}\left(-\hat{\pi}\left(t_{i}+\right)+\hat{\pi}\left(t_{i}-\right)\right)+F^{\prime} \hat{\xi}\left(t_{i}\right)=0, \quad i=1, \ldots, p-1, \\
& E^{\prime}\left(\hat{\pi}\left(t_{p}-\right)\right)+F^{\prime} \hat{\xi}\left(t_{p}\right)=0, \\
& \hat{\eta}\left(t_{i}-\right), \hat{\eta}\left(t_{i-1}+\right) \geq 0, \quad i=1, \ldots, p, \\
& \hat{\xi}\left(t_{i}\right), \hat{\xi}\left(\frac{t_{i-1}+t_{i}}{2}\right) \leq 0, \quad i=1, \ldots, p .
\end{aligned}
$$

Similar to the second discretization in Pullan [41], the importance of $A P(P)$ lies in the fact that feasible solutions for its dual problem $A P^{*}(P)$ can be used either to 
define a feasible solution for $\left(S C S C L P^{*}\right)$ with the same solution value or to define a sequence of feasible solutions for $\left(S C S C L P^{*}\right)$ whose solution value converges to that of the original solution to $A P^{*}(P)$, as shown in the following theorem.

TheOrem 1. Suppose that $P$ is a purified partition of $[0, T]$ (as defined at the end of section 4$)$. Given any feasible solution $(\hat{\pi}, \hat{\eta}, \hat{\xi})$ to $A P^{*}(P)$, if $(9)$ holds for $P$, then there exists a feasible solution $(\pi(t), \eta(t), \xi(t))$ to $\left(S C S C L P^{*}\right)$ whose solution value equals that of $(\hat{\pi}, \hat{\eta}, \hat{\xi})$. Otherwise, there exists a series of feasible solutions $\left(\pi^{k}(t), \eta^{k}(t), \xi^{k}(t)\right)$ to $\left(S C S C L P^{*}\right)$ that are piecewise linear with partition $P^{k}$, whose solution value converges to that of $(\hat{\pi}, \hat{\eta}, \hat{\xi})$ with $P^{k}$ satisfying $(9)$.

Proof. When there are no zero-length intervals in $P$ (i.e., (9) holds), we let

$$
\xi(t)= \begin{cases}\sum_{j=i+1}^{p}\left(\hat{\xi}\left(\frac{t_{j}+t_{j-1}}{2}\right)+\hat{\xi}\left(t_{j}\right)\right) & \text { if } t=t_{i}, i=0,1, \ldots, p-1, \\ 0 & \text { if } t=T .\end{cases}
$$

For $t \in\left(t_{i-1}, t_{i}\right)$, we let

$$
\xi(t)=\frac{t_{i}-t}{t_{i}-t_{i-1}} \xi\left(t_{i-1}\right)+\frac{t-t_{i-1}}{t_{i}-t_{i-1}}\left(\xi\left(t_{i}\right)+\hat{\xi}\left(t_{i}\right)\right) .
$$

We note that $\xi(t)$ is monotonically increasing and right continuous (albeit discontinuous). Let $\pi(t)$ and $\eta(t)$ be the piecewise-linear extensions of $\hat{\pi}$ and $\hat{\eta}$, respectively. It can be shown that $(\pi(t), \eta(t), \xi(t))$ is a feasible solution for $\left(S C S C L P^{*}\right)$ by virtue of the piecewise linearity of the problem data. Now, let us check the relationship between the solution value of the newly constructed solution of $\left(S C S C L P^{*}\right)$ and the original solution of $A P^{*}(P)$. Through integration by parts, we have

$$
\begin{aligned}
& -\int_{0}^{T} a(t)^{\prime} d \pi(t) \\
= & -\left.a(t)^{\prime} \pi(t)\right|_{0} ^{T}+\int_{0}^{T} \pi(t)^{\prime} d a(t) \\
= & \hat{\pi}\left(t_{0}+\right)^{\prime} a\left(t_{0}\right)+\sum_{i=1}^{p}\left(\frac{a\left(t_{i}\right)-a\left(t_{i-1}\right)}{t_{i}-t_{i-1}}\right)^{\prime} \int_{t_{i-1}}^{t_{i}} \pi(t) d t \\
= & \hat{\pi}\left(t_{0}+\right)^{\prime} a\left(t_{0}\right)+\sum_{i=1}^{p}\left(\hat{\pi}\left(t_{i-1}+\right)+\hat{\pi}\left(t_{i}-\right)\right)^{\prime}\left(a\left(t_{i}\right)-a\left(\frac{t_{i}+t_{i-1}}{2}\right)\right) .
\end{aligned}
$$

Since $\eta(t)$ is piecewise linear and $b(t)$ is piecewise constant with partition $P$, we have

$$
\int_{t_{i-1}}^{t_{i}} b(t)^{\prime} \eta(t) d t=\left(\frac{t_{i}-t_{i-1}}{2}\right)\left(\hat{\eta}\left(t_{i-1}+\right)+\hat{\eta}\left(t_{i}-\right)\right)^{\prime} b\left(t_{i}-\right), \quad i=1, \ldots, p .
$$

So

$$
-\int_{0}^{T} b(t)^{\prime} \eta(t) d t=-\sum_{i=1}^{p}\left(\frac{t_{i}-t_{i-1}}{2}\right)\left(\hat{\eta}\left(t_{i-1}+\right)+\hat{\eta}\left(t_{i}-\right)\right)^{\prime} b\left(t_{i}-\right) .
$$

Direct calculation gives

$$
-\int_{0}^{T} h(t)^{\prime} d \xi(t)
$$




$$
\begin{aligned}
= & -\left.h(t)^{\prime} \xi(t)\right|_{0} ^{T}+\int_{0}^{T} \xi(t)^{\prime} d h(t) \\
= & h\left(t_{0}\right)^{\prime} \xi\left(t_{0}\right)+\sum_{i=1}^{p}\left(\frac{h\left(t_{i}\right)-h\left(t_{i-1}\right)}{t_{i}-t_{i-1}}\right)^{\prime} \int_{t_{i-1}}^{t_{i}} \xi(t) d t \\
= & h\left(t_{0}\right)^{\prime} \sum_{j=1}^{p}\left(\hat{\xi}\left(\frac{t_{j}+t_{j-1}}{2}\right)+\hat{\xi}\left(t_{j}\right)\right) \\
& +\sum_{i=1}^{p}\left(\frac{h\left(t_{i}\right)-h\left(t_{i-1}\right)}{2}\right)^{\prime} \\
& \times\left(2 \sum_{j=i+1}^{p}\left(\hat{\xi}\left(\frac{t_{j}+t_{j-1}}{2}\right)+\hat{\xi}\left(t_{j}\right)\right)+\hat{\xi}\left(\frac{t_{i}+t_{i-1}}{2}\right)+2 \hat{\xi}\left(t_{i}\right)\right) \\
= & \sum_{i=1}^{p}\left(\hat{\xi}\left(t_{i}\right)^{\prime} h\left(t_{i}\right)+\hat{\xi}\left(\frac{t_{i-1}+t_{i}}{2}\right)^{\prime} h\left(\frac{t_{i-1}+t_{i}}{2}\right)\right) .
\end{aligned}
$$

Combining (15), (16), and (17), we see that $(\pi(t), \eta(t), \xi(t))$ has the same solution value as $(\hat{\pi}, \hat{\eta}, \hat{\xi})$. This proves the first part of the theorem.

Now, suppose (9) does not hold for $P$. Since $P$ is a purified partition, by Corollary 2 and Lemma 4, the zero-length intervals in $P$ can be located only at the breakpoints in $D_{1}^{P}$. So for any zero-length interval $\left[t_{i-1}, t_{i}\right]$ that resides on $\left[t_{l}, t_{m}\right]$, where $t_{l}$ and $t_{m}$ are two consecutive breakpoints in $D_{1}^{P}$, either $t_{i-1}=t_{l}$ or $t_{i}=t_{m}$. Let $\tau \in(0,1)$. We define a new solution $\left(\tilde{\pi}^{\tau}, \tilde{\eta}^{\tau}, \tilde{\xi}^{\tau}\right)$ in the following way.

If $t_{i-1}=t_{l}$, we let

$$
\begin{aligned}
\tilde{t}_{i-1}^{\tau} & =t_{i-1}, \\
\tilde{t}_{i}^{\tau} & =(1-\tau) t_{i}+\tau t_{i+1}, \\
\tilde{t}_{i+1}^{\tau} & =t_{i+1}, \\
\tilde{\pi}^{\tau}\left(\tilde{t}_{i}^{\tau}-\right) & =(1-\tau) \hat{\pi}\left(t_{i}-\right)+\tau \hat{\pi}\left(t_{i}+\right), \\
\tilde{\pi}^{\tau}\left(\tilde{t}_{i}^{\tau}+\right) & =(1-\tau) \hat{\pi}\left(t_{i}+\right)+\tau \hat{\pi}\left(t_{i+1}-\right), \\
\tilde{\eta}^{\tau}\left(\tilde{t}_{i}^{\tau}-\right) & =(1-\tau) \hat{\eta}\left(t_{i}-\right)+\tau \hat{\eta}\left(t_{i}+\right), \\
\tilde{\eta}^{\tau}\left(\tilde{t}_{i}^{\tau}+\right) & =(1-\tau) \hat{\eta}\left(t_{i}+\right)+\tau \hat{\eta}\left(t_{i+1}-\right), \\
\tilde{\xi}^{\tau}\left(\frac{\tilde{t}_{i}^{\tau}+\tilde{t}_{i-1}^{\tau}}{2}\right) & =(1-\tau) \hat{\xi}\left(\frac{t_{i}+t_{i-1}}{2}\right)+\tau \hat{\xi}\left(t_{i}\right), \\
\tilde{\xi}^{\tau}\left(\frac{\tilde{t}_{i}^{\tau}+\tilde{t}_{i+1}^{\tau}}{2}\right) & =(1-\tau) \hat{\xi}\left(\frac{t_{i}+t_{i+1}}{2}\right) .
\end{aligned}
$$

If $t_{i}=t_{m}$, we let

$$
\begin{aligned}
\tilde{t}_{i-2}^{\tau} & =t_{i-2} \\
\tilde{t}_{i-1}^{\tau} & =(1-\tau) t_{i-1}+\tau t_{i-2}, \\
\tilde{t}_{i}^{\tau} & =t_{i} \\
\tilde{\pi}^{\tau}\left(\tilde{t}_{i-1}^{\tau}-\right) & =(1-\tau) \hat{\pi}\left(t_{i-1}-\right)+\tau \hat{\pi}\left(t_{i-2}+\right),
\end{aligned}
$$




$$
\begin{aligned}
\tilde{\pi}^{\tau}\left(\tilde{t}_{i-1}^{\tau}+\right) & =(1-\tau) \hat{\pi}\left(t_{i-1}+\right)+\tau \hat{\pi}\left(t_{i-1}-\right), \\
\tilde{\eta}^{\tau}\left(\tilde{t}_{i-1}^{\tau}-\right) & =(1-\tau) \hat{\eta}\left(t_{i-1}-\right)+\tau \hat{\eta}\left(t_{i-2}+\right), \\
\tilde{\eta}^{\tau}\left(\tilde{t}_{i-1}^{\tau}+\right) & =(1-\tau) \hat{\eta}\left(t_{i-1}+\right)+\tau \hat{\eta}\left(t_{i-1}-\right), \\
\tilde{\xi}^{\tau}\left(\frac{\tilde{t}_{i}^{\tau}+\tilde{t}_{i-1}^{\tau}}{2}\right) & =(1-\tau) \hat{\xi}\left(\frac{t_{i}+t_{i-1}}{2}\right)+\tau \hat{\xi}\left(t_{i-1}\right), \\
\tilde{\xi}^{\tau}\left(\tilde{t}_{i-1}^{\tau}\right) & =(1-\tau) \hat{\xi}\left(t_{i-1}\right)+\tau \hat{\xi}\left(\frac{t_{i-1}+t_{i-2}}{2}\right), \\
\tilde{\xi}^{\tau}\left(\frac{\tilde{t}_{i-1}^{\tau}+\tilde{t}_{i-2}^{\tau}}{2}\right) & =(1-\tau) \hat{\xi}\left(\frac{t_{i-1}+t_{i-2}}{2}\right) .
\end{aligned}
$$

For all the other quantities not defined in the above cases, we let $\tilde{t}_{j}^{\tau}=t_{j}$, $\tilde{\pi}^{\tau}\left(\tilde{t}_{j}^{\tau}-\right)=\hat{\pi}\left(t_{j}-\right), \tilde{\pi}^{\tau}\left(\tilde{t}_{j}^{\tau}+\right)=\hat{\pi}\left(t_{j}+\right), \tilde{\eta}^{\tau}\left(\tilde{t}_{j}^{\tau}-\right)=\hat{\eta}\left(t_{j}-\right), \tilde{\eta}^{\tau}\left(\tilde{t}_{j}^{\tau}+\right)=\hat{\eta}\left(t_{j}+\right), \tilde{\xi}^{\tau}\left(\tilde{t}_{j-1}^{\tau}\right)=$ $\hat{\xi}\left(t_{j-1}\right)$, and $\tilde{\xi}^{\tau}\left(\frac{\tilde{t}_{j}^{\tau}+\tilde{t}_{j-1}^{\tau}}{2}\right)=\hat{\xi}\left(\frac{t_{j}+t_{j-1}}{2}\right)$.

Let $P^{\tau}$ be the partition defined from $\tilde{t}^{\tau}$. It is easy to check the feasibility of $\left(\tilde{\pi}^{\tau}, \tilde{\eta}^{\tau}, \tilde{\xi}^{\tau}\right)$ to $A P^{*}\left(P^{\tau}\right)$. Since $\left(\tilde{\pi}^{\tau}, \tilde{\eta}^{\tau}, \tilde{\xi}^{\tau}\right)$ converges to $(\hat{\pi}, \hat{\eta}, \hat{\xi})$ and $\tilde{t}^{\tau}$ converges to $\hat{t}$ as $\tau$ tends to zero, we see that the solution value of $\left(\tilde{\pi}^{\tau}, \tilde{\eta}^{\tau}, \tilde{\xi}^{\tau}\right)$ in $A P^{*}\left(P^{\tau}\right)$ converges to the solution value of $(\hat{\pi}, \hat{\eta}, \hat{\xi})$ in $A P^{*}(P)$. Furthermore, (9) holds for $P^{\tau}$. Applying the first part of the theorem to $P^{\tau}$, we conclude that the theorem is true for $P$.

We may now summarize the relationship among the values of various discrete approximations in the following theorem (see also Theorem 3.5 in Pullan [41]).

Theorem 2. For any partitions $P$ and $Q$,

$$
V(A P(P))=V\left(A P^{*}(P)\right) \leq V\left(\left(S C S C L P^{*}\right)\right) \leq V((S C S C L P)) \leq V(D P(Q)) .
$$

Proof. By the strong duality result for finite-dimensional linear programming, the value of the optimal solution to $A P(P)$ is the value of the optimal solution to its dual $A P^{*}(P)$. By Theorem 1, the solution value of this solution can be closely approximated by a sequence of feasible solutions to $\left(S C S C L P^{*}\right)$. It then follows that this value is a lower bound on $V\left(\left(S C S C L P^{*}\right)\right)$, and thus a lower bound on $V((S C S C L P))$ by Proposition 1. The final inequality follows from the definition of $D P(Q)$.

Corollary 3. For any partitions $P$ and $Q$, if

$$
V(A P(P)) \geq V(Q P(|Q|)),
$$

then the optimal solution value of $Q P(|Q|)$ gives the optimal solution value to $(S C S C L P)$. In particular, if a solution $(\hat{v}, \hat{y}, \hat{t})$ is feasible for $Q P(|Q|)$ and has the same cost as the optimal value of $A P(P)$, then $(\hat{v}, \hat{y}, \hat{t})$ gives the optimal solution value for (SCSCLP) which can be closely approximated by a sequence of feasible solutions to $(S C S C L P)$.

Proof. By Lemma 2, the solution value of any feasible solution to $Q P(|Q|)$ is an upper bound on $V((S C S C L P))$, and the result follows directly from Theorem 2 .

5.2. The doubling of breakpoints. Based on a new discrete approximation of $(S C L P)$ similar to $A P 1(P)$, Pullan [41] found a descent solution for $(S C L P)$ (consequently, a descent direction can be constructed) by patching together the current 
solution and a solution that has a better solution value in $A P 1(P)$ than the current solution. The new solution has a strictly improved solution value in $(S C L P)$ but usually has three times as many constant control pieces as the original solution. In the following, we give a construction for a feasible solution to $(S C S C L P)$ that produces, at most, approximately twice as many breakpoints as the original feasible solution.

Let $P$ be a partition of $[0, T]$, and define a new partition as follows:

$$
\bar{P}=\left\{t_{0}, t_{0}, t_{1}, t_{1}, \ldots, t_{i}, t_{i}, t_{i}, \ldots, t_{p}, t_{p}\right\},
$$

where each breakpoint in $D^{P}$ has two duplicates and all the other breakpoints have only one duplicate. Intuitively, we have placed a zero-length interval at the beginning of every breakpoint of $P$ and put a zero-length interval at the end of each breakpoint in $D^{P}$. Under this configuration, the set of intervals in $\bar{P}$ is the union of the intervals in $P$ and a set of zero-length intervals. We let $\bar{t}_{i}$ denote the $(i+1)$ th element of $\bar{P}$. $D_{1}^{\bar{P}}$ is the set of breakpoints in $\bar{P}$ that correspond to the breakpoints in $D_{1}^{P}$. For the $i$ th interval (i.e., $\left.\left[t_{i-1}, t_{i}\right]\right)$ in $P$, we have a corresponding interval $\left[\bar{t}_{j-1}, \bar{t}_{j}\right]$ in $\bar{P}$, where $\bar{t}_{j-1}=t_{i-1}$ and $\bar{t}_{j}=t_{i}$. We call this interval in $\bar{P}$ an old interval. All the other intervals in $\bar{P}$ are called new intervals. Note that all the new intervals have zero length but not vice versa.

Given a solution $(\hat{v}, \hat{y}, \hat{t})$ to $Q P(|P|)$, we first construct a feasible solution $(\bar{v}, \bar{y}, \bar{t})$ to $Q P(|\bar{P}|)$ and then show a descent direction for this solution in $Q P(|\bar{P}|)$. The descent direction will be used in the proof of convergence. We need not use the same direction in the new algorithm, as we will see in the last remark in section 6 . This solution has the same solution value in $Q P(|\bar{P}|)$ as the current solution in $Q P(|P|)$ and has approximately twice as many intervals, fewer than the one constructed by Pullan [41].

Let $(\hat{v}, \hat{y}, \hat{t})$ be a feasible solution for $Q P(|P|)$. For the $i$ th interval in $\bar{P}$, if it is an old interval, we let interval $j$ be the corresponding interval in $P$ and set

$$
\bar{v}\left(\bar{t}_{i}\right)=\hat{v}\left(t_{j}\right), \quad \bar{y}\left(\bar{t}_{i}\right)=\hat{y}\left(t_{j}\right) .
$$

We let $\bar{v}\left(\bar{t}_{i}\right)=0$ if interval $i$ in $\bar{P}$ is a new interval and let $\bar{y}\left(\bar{t}_{i}\right)=\hat{y}\left(t_{j}\right)$, where $j$ is the interval in $P$ that corresponds to the closest old interval in $\bar{P}$ to the left of $\left[t_{i-1}, t_{i}\right]$ (with the convention that $\bar{y}\left(\bar{t}_{1}\right)=y\left(t_{0}\right)$ and $\bar{y}\left(\bar{t}_{0}\right)=y\left(t_{0}\right)$ ).

It is easy to verify that $(\bar{v}, \bar{y}, \bar{t})$ is feasible for $Q P(|\bar{P}|)$ and has the same solution value in $Q P(|\bar{P}|)$ as $(\hat{v}, \hat{y}, \hat{t})$ in $Q P(|P|)$.

5.3. A descent direction. According to Corollary 3 , a feasible solution $(\hat{v}, \hat{y}, \hat{t})$ to $Q P(|P|)$ gives the optimal solution value of $(S C S C L P)$ if the optimal solution to $A P(P)$ has an equal or larger solution value. If so, we can stop the algorithm. Otherwise, there exists $(\tilde{\bar{v}}, \tilde{\bar{y}}, \tilde{t})$ feasible for $A P(P)$ and with a strictly smaller solution value in $A P(P)$, i.e., we have

$$
\delta \stackrel{\text { def }}{=} V(A P(P),(\tilde{\bar{v}}, \tilde{\bar{y}}, \tilde{\bar{t}}))-V(Q P(|P|),(\hat{v}, \hat{y}, \hat{t}))<0 .
$$

Note that $|\delta|$ is an upper bound on the duality gap between (SCSCLP) and (SCSCLP*).

Let $\epsilon \in[0,1]$. For every interval $\left[t_{i-1}, t_{i}\right]$, we define

$$
\epsilon_{i}=\frac{\left(t_{i}-t_{i-1}\right) \epsilon}{2} \text {. }
$$

We define a new partition $P^{\epsilon}$ of $[0, T]$ as follows:

$$
P^{\epsilon} \stackrel{\text { def }}{=}\left\{t_{0}, t_{0}+\epsilon_{1}, t_{1}-\epsilon_{1}, t_{1}+\epsilon_{2}, \ldots, t_{i}-\epsilon_{i}, t_{i}, t_{i}+\epsilon_{i+1}, \ldots, t_{p}-\epsilon_{p}, t_{p}\right\},
$$




\begin{tabular}{|c|c|}
\hline$\widetilde{\bar{u}}(t)$ & $t_{l}$ \\
\hline$\hat{u}(t)$ & \\
\hline$\hat{\bar{u}}(t)$ & $t_{l+1}$ \\
\hline : & $t_{l+1}-\varepsilon_{l+2}$ \\
\hline$\hat{\bar{u}}(t)$ & $\begin{array}{l}t_{i-1}-\varepsilon_{i-1} \\
t_{i-1}\end{array}$ \\
\hline$\hat{u}(t)$ & ${ }_{i-1}+$ \\
\hline$\vdots$ & $i$. \\
\hline$\hat{\bar{u}}(t)$ & $\begin{array}{l}t_{m-1}-\varepsilon_{m-1} \\
t_{m-1}\end{array}$ \\
\hline$\hat{u}(t)$ & \\
\hline$\widetilde{\bar{u}}(t)$ & $t_{m}-\varepsilon_{m}$ \\
\hline
\end{tabular}

FIG. 2. The construction of a descent solution where $t_{l}$ and $t_{m}$ are two consecutive breakpoints in $D_{1}$.

where we replace the breakpoint $t_{i}$ in $P \backslash D_{1}^{P}$ with two elements $t_{i}-\epsilon_{i}$ and $t_{i}+\epsilon_{i+1}$ and add two elements $t_{i}-\epsilon_{i}$ and $t_{i}+\epsilon_{i+1}$. For breakpoint $t_{i}$ in $D^{P}$, we add $t_{0}+\epsilon_{1}$ and $t_{p}-\epsilon_{p}$ for $t_{0}$ and $t_{p}$, respectively. We define the vector $t^{\epsilon}$ from $P^{\epsilon}$ by mapping $t_{i}^{\epsilon}$ to the $(i+1)$ th element in $P^{\epsilon}$. We construct a descent solution $\left(v^{\epsilon}, y^{\epsilon}\right.$, $\left.t^{\epsilon}\right)$ with partition $P^{\epsilon}$ as follows.

When $P$ does not have any zero-length intervals, let $\tilde{\bar{u}}(t), \hat{u}(t)$, and $\hat{\bar{u}}(t)$ be the piecewise constant extensions of $\tilde{\bar{u}}, \hat{u}$, and $\hat{\bar{u}}$, respectively, where $\tilde{\bar{u}}$ is defined from $\tilde{\bar{v}}$ by

$$
\tilde{\bar{u}}\left(t_{i-1}+\right)=2 \frac{\tilde{\bar{v}}\left(t_{i-1}+\right)}{t_{i}-t_{i-1}}, \quad \tilde{\bar{u}}\left(t_{i}-\right)=2 \frac{\tilde{\bar{v}}\left(t_{i}-\right)}{t_{i}-t_{i-1}}
$$

$\hat{u}$ is defined from $\hat{v}$ by (8), and $\hat{\bar{u}}$ is defined as

$$
\hat{\bar{u}}\left(t_{i-1}+\right)=\frac{\tilde{\bar{v}}\left(t_{i-1}+\right)+\tilde{\bar{v}}\left(t_{i}-\right)}{t_{i}-t_{i-1}} .
$$

We construct the new control by patching together $\tilde{\bar{u}}(t), \hat{u}(t)$, and $\hat{\bar{u}}(t)$ as follows:

$$
u^{\epsilon}(t)= \begin{cases}\tilde{\bar{u}}(t), & t \in\left[t_{i-1}, t_{i-1}+\epsilon_{i}\right) \bigcup\left[t_{i}-\epsilon_{i}, t_{i}\right), t_{i} \in D^{P} \\ \tilde{\bar{u}}(t), & t \in\left[t_{p-1}, t_{p-1}+\epsilon_{p}\right) \bigcup\left[t_{p}-\epsilon_{p}, t_{p}\right] \\ \hat{u}(t), & t \in\left[t_{i-1}+\epsilon_{i}, t_{i}-\epsilon_{i}\right) \\ \hat{\bar{u}}(t), & \text { otherwise. }\end{cases}
$$

Having constructed the control, the construction of the state variables for (SCSCLP) is straightforward. Our construction of a descent solution $\left(v^{\epsilon}, y^{\epsilon}, t^{\epsilon}\right)$ for $(\hat{v}, \hat{y}, \hat{t})$ is illustrated in Figure 2.

However, if $t_{i-1}=t_{i}$ for some $i$, the $u$ variables in the previous paragraph are not properly defined. Fortunately, we can bypass this difficulty by working on the $v$ variables. We define $v^{\epsilon}$ as follows. Let $t_{l}$ and $t_{m}$ be two consecutive breakpoints in 
$D_{1}^{P}$. Let $\left[t_{i}+\epsilon_{i+1}, t_{i+1}-\epsilon_{i+1}\right]$ and $\left[t_{i+1}-\epsilon_{i+1}, t_{i+1}+\epsilon_{i+2}\right]$ be two intervals that reside on $\left[t_{l}, t_{m}\right]$. If $t_{j}^{\epsilon}$ is the breakpoint in $P^{\epsilon}$ that is mapped to $t_{i+1}-\epsilon_{i+1}$, we let

$$
\begin{aligned}
v^{\epsilon}\left(t_{j}^{\epsilon}\right) & =(1-\epsilon) \hat{v}\left(t_{i+1}\right), \\
v^{\epsilon}\left(t_{j+1}^{\epsilon}\right) & =\epsilon\left(\tilde{\bar{v}}\left(t_{i+1}-\right)+\tilde{v}\left(t_{i+1}+\right)\right) .
\end{aligned}
$$

If $t_{j}^{\epsilon}$ is the breakpoint in $P^{\epsilon}$ that is mapped to $t_{l}$, we let

$$
v^{\epsilon}\left(t_{j+1}^{\epsilon}\right)=\epsilon \tilde{\bar{v}}\left(t_{l}+\right) .
$$

If $t_{j}^{\epsilon}$ is the breakpoint in $P^{\epsilon}$ that is mapped to $t_{m}$, we let

$$
v^{\epsilon}\left(t_{j}^{\epsilon}\right)=\epsilon \tilde{\bar{v}}\left(t_{m}-\right) .
$$

We define $y^{\epsilon}$ in three different cases as follows. For the breakpoint $t_{j}^{\epsilon}$ in $P^{\epsilon}$ that is mapped to $t_{i-1}+\epsilon_{i}$, we let

$$
y^{\epsilon}\left(t_{j}^{\epsilon}\right)=(1-\epsilon) \hat{y}\left(t_{i-1}\right)+\epsilon \tilde{\bar{y}}\left(\frac{t_{i}+t_{i-1}}{2}\right) .
$$

For the breakpoint $t_{j}^{\epsilon}$ in $P^{\epsilon}$ that is mapped to $t_{i}-\epsilon_{i}$, we let

$$
y^{\epsilon}\left(t_{j}^{\epsilon}\right)=(1-\epsilon) \hat{y}\left(t_{i}\right)+\epsilon \tilde{\bar{y}}\left(\frac{t_{i}+t_{i-1}}{2}\right) .
$$

For the breakpoint $t_{j}^{\epsilon}$ in $P^{\epsilon}$ that is mapped to $t_{i}$, we let

$$
y^{\epsilon}\left(t_{j}^{\epsilon}\right)=(1-\epsilon) \hat{y}\left(t_{i}\right)+\epsilon \tilde{\bar{y}}\left(t_{i}\right) .
$$

When $\epsilon$ is small, $\left(v^{\epsilon}, y^{\epsilon}, t^{\epsilon}\right)$ is a descent solution as shown in the following theorem.

THeOREM 3. If (19) holds, then $\left(v^{\epsilon}, y^{\epsilon}, t^{\epsilon}\right)$ is a feasible solution to $Q P(|\bar{P}|)$ and

$$
V\left(Q P(|\bar{P}|),\left(v^{\epsilon}, y^{\epsilon}, t^{\epsilon}\right)\right)-V(Q P(|\bar{P}|),(\bar{v}, \bar{y}, \bar{t}))=\epsilon \delta+o(\epsilon),
$$

where $\delta$ is defined in (19). For $\epsilon$ small enough, $\left(v^{\epsilon}, y^{\epsilon}, t^{\epsilon}\right)$ has a strictly smaller solution value than $(\bar{v}, \bar{y}, \bar{t})$.

Proof. The feasibility of $\left(v^{\epsilon}, y^{\epsilon}, t^{\epsilon}\right)$ follows easily. By definition, we have

$$
\begin{aligned}
V(Q P(|\bar{P}|),(\bar{v}, \bar{y}, \bar{t})) & =V(Q P(|P|),(\hat{v}, \hat{y}, \hat{t})) \\
& =\sum_{i=1}^{p} \hat{v}\left(t_{i}\right)^{\prime} c\left(\frac{t_{i}+t_{i-1}}{2}\right)+\sum_{i=1}^{p} \frac{t_{i}-t_{i-1}}{2}\left(\hat{y}\left(t_{i}\right)\right. \\
& \left.+\hat{y}\left(t_{i-1}\right)\right)^{\prime} g\left(t_{i-1}+\right), \\
V\left(Q P(|\bar{P}|),\left(v^{\epsilon}, y^{\epsilon}, t^{\epsilon}\right)\right) & =\sum_{i=1}^{\left|P^{\epsilon}\right|-1} c\left(\frac{t_{i}^{\epsilon}+t_{i-1}^{\epsilon}}{2}\right)^{\prime} v^{\epsilon}\left(t_{i}^{\epsilon}\right) \\
& +\sum_{i=1}^{\left|P^{\epsilon}\right|-1} \frac{t_{i}^{\epsilon}-t_{i-1}^{\epsilon}}{2}\left(y^{\epsilon}\left(t_{i}^{\epsilon}\right)+y^{\epsilon}\left(t_{i-1}^{\epsilon}\right)\right)^{\prime} g\left(t_{i-1}^{\epsilon}+\right) .
\end{aligned}
$$

Let $t_{l}$ and $t_{m}$ be two consecutive breakpoints in $D_{1}^{P}$ and let $t_{\bar{l}}^{\epsilon}$ and $t_{\bar{m}}^{\epsilon}$ be the corresponding breakpoints in $D_{1}^{P^{\epsilon}}$. We have

$$
\sum_{i=l+1}^{m} \hat{v}\left(t_{i}\right)^{\prime} c\left(\frac{t_{i}+t_{i-1}}{2}\right)-\sum_{i=\bar{l}+1}^{\bar{m}} c\left(\frac{t_{i}^{\epsilon}+t_{i-1}^{\epsilon}}{2}\right)^{\prime} v^{\epsilon}\left(t_{i}^{\epsilon}\right)
$$




$$
\begin{aligned}
= & \sum_{i=l+1}^{m} \hat{v}\left(t_{i}\right)^{\prime} c\left(\frac{t_{i}+t_{i-1}}{2}\right)-\sum_{i=l+1}^{m}(1-\epsilon) \hat{v}\left(t_{i}\right)^{\prime} c\left(\frac{t_{i}+t_{i-1}}{2}\right) \\
& -\sum_{i=l+1}^{m-1} \epsilon\left(\tilde{\bar{v}}\left(t_{i}+\right)+\tilde{\bar{v}}\left(t_{i}-\right)\right)^{\prime} c\left(t_{i}+\frac{\epsilon_{i+1}-\epsilon_{i}}{2}\right) \\
& -\epsilon \tilde{\bar{v}}\left(t_{l}+\right)^{\prime} c\left(t_{l}+\frac{\epsilon_{i+1}}{2}\right)-\epsilon \tilde{\bar{v}}\left(t_{m}-\right)^{\prime} c\left(t_{m}-\frac{\epsilon_{m}}{2}\right) \\
= & \sum_{i=l+1}^{m} \epsilon\left(\hat{v}\left(t_{i}\right)^{\prime} c\left(\frac{t_{i}+t_{i-1}}{2}\right)-\left(\tilde{\bar{v}}\left(t_{i-1}+\right)^{\prime} c\left(t_{i-1}+\right)+\tilde{\bar{v}}\left(t_{i}-\right)^{\prime} c\left(t_{i}-\right)\right)\right)+o(\epsilon),
\end{aligned}
$$

and

$$
\begin{aligned}
& \sum_{i=l+1}^{m} \frac{t_{i}-t_{i-1}}{2}\left(\hat{y}\left(t_{i}\right)+\hat{y}\left(t_{i-1}\right)\right)^{\prime} g\left(t_{i-1}+\right)-\sum_{i=\bar{l}+1}^{\bar{m}} \frac{t_{i}^{\epsilon}-t_{i-1}^{\epsilon}}{2}\left(y^{\epsilon}\left(t_{i}^{\epsilon}\right)+y^{\epsilon}\left(t_{i-1}^{\epsilon}\right)\right)^{\prime} g\left(t_{i-1}^{\epsilon}+\right) \\
= & \sum_{i=l+1}^{m} \frac{t_{i}-t_{i-1}}{2}\left(\hat{y}\left(t_{i}\right)+\hat{y}\left(t_{i-1}\right)\right)^{\prime} g\left(t_{i-1}+\right) \\
& -\sum_{i=l+1}^{m}(1-\epsilon) \frac{t_{i}-t_{i-1}}{2}\left((1-\epsilon)\left(\hat{y}\left(t_{i}\right)+\hat{y}\left(t_{i-1}\right)\right)+2 \epsilon \tilde{\bar{y}}\left(\frac{t_{i}+t_{i-1}}{2}\right)\right)^{\prime} g\left(t_{i-1}+\right) \\
& -\sum_{i=l+1}^{m} \frac{\epsilon_{i}+\epsilon_{i+1}}{2}\left(2(1-\epsilon) \hat{y}\left(t_{i}\right)+\epsilon\left(\tilde{\tilde{y}}\left(\frac{t_{i}+t_{i-1}}{2}\right)+\tilde{\bar{y}}\left(\frac{t_{i+1}+t_{i}}{2}\right)\right)^{\prime} g\left(t_{i-1}+\right)\right. \\
& -\frac{\epsilon_{l+1}}{2}\left(2(1-\epsilon) \hat{y}\left(t_{l}\right)+\epsilon\left(\tilde{\bar{y}}\left(t_{l}\right)+\tilde{\bar{y}}\left(\frac{t_{l+1}+t_{l}}{2}\right)\right)\right)^{\prime} g\left(t_{l}+\right) \\
& -\frac{\epsilon_{m}}{2}\left(2(1-\epsilon) \hat{y}\left(t_{m}\right)+\epsilon\left(\tilde{\bar{y}}\left(t_{m}\right)+\tilde{\bar{y}}\left(\frac{t_{m-1}+t_{m}}{2}\right)\right)\right)^{\prime} g\left(t_{m}-\right) \\
= & \sum_{i=l+1}^{m} \epsilon \frac{t_{i}-t_{i-1}}{2}\left(\hat{y}\left(t_{i}\right)+\hat{y}\left(t_{i-1}\right)\right)^{\prime} g\left(t_{i-1}+\right) \\
& -\sum_{i=l+1}^{m}\left(t_{i}-t_{i-1}\right) \epsilon \tilde{\bar{y}}\left(\frac{t_{i}+t_{i-1}}{2}\right)^{\prime} g\left(t_{i-1}+\right)+o(\epsilon) .
\end{aligned}
$$
have

Summing up (28) and (29) over all pairs of consecutive breakpoints in $D_{1}$, we

$$
\begin{aligned}
& V\left(Q P(|\bar{P}|),\left(v^{\epsilon}, y^{\epsilon}, t^{\epsilon}\right)\right)-V(Q P(|\bar{P}|),(\bar{v}, \bar{y}, \bar{t})) \\
= & V\left(Q P(|\bar{P}|),\left(v^{\epsilon}, y^{\epsilon}, t^{\epsilon}\right)\right)-V(Q P(|P|),(\hat{v}, \hat{y}, \hat{t})) \\
= & \sum_{i=1}^{p} \epsilon\left(\tilde{\tilde{v}}\left(t_{i-1}+\right)^{\prime} c\left(t_{i-1}+\right)+\tilde{\bar{v}}\left(t_{i}-\right)^{\prime} c\left(t_{i}-\right)-\hat{v}\left(t_{i}\right)^{\prime} c\left(\frac{t_{i}+t_{i-1}}{2}\right)\right) \\
& \left.\quad-\frac{t_{i}-t_{i-1}}{2}\left(\hat{y}\left(t_{i}\right)+\hat{y}\left(t_{i-1}\right)\right)^{\prime} g\left(t_{i-1}+\right)\right)+o(\epsilon) \\
& =\epsilon(V(A P(P),(\tilde{\bar{v}}, \tilde{\bar{y}}, \tilde{t}))-V(Q P(|P|),(\hat{v}, \hat{y}, \hat{t})))+o(\epsilon) \\
= & \epsilon \delta+o(\epsilon) .
\end{aligned}
$$


Since $\delta<0$, when $\epsilon$ is small enough, $\left(v^{\epsilon}, y^{\epsilon}, t^{\epsilon}\right)$ is a strictly improved feasible solution to $Q P(|\bar{P}|)$.

Interestingly, the new solution $\left(v^{\epsilon}, y^{\epsilon}, t^{\epsilon}\right)$ gives a descent direction for $(\bar{v}, \bar{y}, \bar{t})$ in $Q P(|\bar{P}|)$. This solution can also be used to show that the first Frank-Wolfe iterate for $(\bar{v}, \bar{y}, \bar{t})$ provides an upper bound on the current duality gap, as we next illustrate.

Let $\left[t_{l}, t_{m}\right]$ be two consecutive breakpoints in $D_{1}^{P}$. We define a new partition $\overline{\bar{P}}$ as follows. The set of breakpoints of $\overline{\bar{P}}$ that resides on $\left[t_{l}, t_{m}\right]$ is $\left\{t_{l}, \frac{t_{l}+t_{l+1}}{2}, \frac{t_{l}+t_{l+1}}{2}, \ldots, t_{m}\right\}$, i.e., the union of $\left\{t_{l}, t_{m}\right\}$ with the set of midpoints of the intervals in $P$, and each midpoint appears exactly twice. We construct $(\overline{\bar{v}}, \overline{\bar{y}}, \overline{\bar{t}})$ as follows. The set of breakpoints of $(\overline{\bar{v}}, \overline{\bar{y}}, \overline{\bar{t}})$ is $\overline{\bar{P}}$. Let

$$
\overline{\bar{v}}_{j}= \begin{cases}\tilde{\bar{v}}\left(t_{i+1}-\right)+\tilde{\bar{v}}\left(t_{i+1}+\right) & \text { if the } j \text { th interval of } \overline{\bar{P}} \text { is }\left[\frac{t_{i}+t_{i+1}}{2}, \frac{t_{i+1}+t_{i+2}}{2}\right], \\ \tilde{\bar{v}}\left(t_{l}+\right) & \text { if the } j \text { th interval of } \overline{\bar{P}} \text { is }\left[t_{l}, \frac{t_{l}+t_{l+1}}{2}\right], \\ \tilde{\bar{v}}\left(t_{m}-\right) & \text { if the } j \text { th interval of } \overline{\bar{P}} \text { is }\left[\frac{t_{m-1}+t_{m}}{2}, t_{m}\right], \\ 0 & \text { otherwise, }\end{cases}
$$

$\overline{\bar{y}}\left(\overline{\bar{t}}_{0}\right)=y\left(t_{0}\right)$, and

$$
\overline{\bar{y}}\left(\overline{\bar{t}}_{j}\right)= \begin{cases}\tilde{\bar{y}}\left(\frac{t_{i+1}+t_{i+2}}{2}\right) & \text { if the } j \text { th interval of } \overline{\bar{P}} \text { is }\left[\frac{t_{i}+t_{i+1}}{2}, \frac{t_{i+1}+t_{i+2}}{2}\right], \\ \tilde{\bar{y}}\left(\frac{t_{l}+t_{l+1}}{2}\right) & \text { if the } j \text { th interval of } \overline{\bar{P}} \text { is }\left[t_{l}, \frac{t_{l}+t_{l+1}}{2}\right], \\ \tilde{\bar{y}}\left(t_{m}\right) & \text { if the } j \text { th interval of } \overline{\bar{P}} \text { is }\left[\frac{t_{m-1}+t_{m}}{2}, t_{m}\right], \\ \tilde{\bar{y}}\left(\frac{t_{i+1}+t_{i}}{2}\right) & \text { if the } j \text {-th interval of } \overline{\bar{P}} \text { is }\left[\frac{t_{i}+t_{i+1}}{2}, \frac{t_{i}+t_{i+1}}{2}\right] .\end{cases}
$$

Theorem 4. For $\epsilon \in[0,1]$, let $t^{\epsilon}$ be defined by $P^{\epsilon}$. Let $\left(v^{\epsilon}, y^{\epsilon}, t^{\epsilon}\right)$ be the solution to $Q P(|\bar{P}|)$ defined by $(21)-(26)$. We have

$$
\begin{aligned}
v^{\epsilon} & =\epsilon \overline{\bar{v}}+(1-\epsilon) \bar{v} \\
y^{\epsilon} & =\epsilon \overline{\bar{y}}+(1-\epsilon) \bar{y} \\
t^{\epsilon} & =\epsilon \overline{\bar{t}}+(1-\epsilon) \bar{t}
\end{aligned}
$$

and $(\overline{\bar{v}}, \overline{\bar{y}}, \overline{\bar{t}})$ is feasible for $Q P(|\bar{P}|)$.

Proof. Theorem 4 obtains the direct consequence of the definition of $\left(v^{\epsilon}, y^{\epsilon}, t^{\epsilon}\right)$ and $(\overline{\bar{v}}, \overline{\bar{y}}, \overline{\bar{t}})$.

If we pick $(\tilde{\bar{v}}, \tilde{\bar{y}}, \tilde{\bar{t}})$ introduced in (19) as an optimal solution for $A P(P)$, then by Theorem $2,|\delta|$ is an upper bound on the current duality gap. By (27) and Theorem 4 , the negative objective value of the first Frank-Wolfe iterate for $(\bar{v}, \bar{y}, \bar{t})$ gives an upper bound on the current duality gap.

6. A new algorithm for (SCSCLP). In this section, we give a generic successive quadratic programming algorithm for $(S C S C L P)$.

Algorithm $\mathcal{A}(E, F, G, H, a(t), b(t), c(t), g(t), h(t), T, \beta)$.

Let $k=0$. Let $d$ be the current duality gap initially set to infinity.

Let $\left(v^{k}, y^{k}, t^{k}\right)$ be a feasible solution to $Q P\left(\left|P^{0}\right|\right)$. Let $P^{0}$ be a partition on $[0, T]$, such that $a(t), c(t)$, and $h(t)$ are piecewise linear with $P^{0}$ and $b(t)$ and $g(t)$ are piecewise constant with $P^{0}$.

while $d>\beta$ do

1. Calculate a KKT point of $Q P\left(\left|P^{k}\right|\right)$ which has an equal or better solution value than $\left(v^{k}, y^{k}, t^{k}\right)$. 
2. Recursively remove redundant intervals in $P^{k}$ as follows.

Apply Procedure PURIFY to all pairs of consecutive breakpoints in $D_{1}^{P^{k}}$. Let $\left(\tilde{v}^{k}, \tilde{y}^{k}, \tilde{t}^{k}\right)$ be the resulting solution and let $Q$ be the resulting partition. If $\left(\tilde{v}^{k}, \tilde{y}^{k}, \tilde{t}^{k}\right)$ is not a KKT point of $Q P(|Q|)$, let $\left(v^{k}, y^{k}, t^{k}\right)=\left(\tilde{v}^{k}, \tilde{y}^{k}, \tilde{t}^{k}\right)$ and $P^{k}=Q$ and go to Step 1. Otherwise, we denote the resulting purified partition as $\tilde{P}^{k}=\left\{t_{0}, t_{1}, \ldots, t_{p}\right\}$.

3. Double the number of intervals. Define $P^{k+1}$ as

$$
P^{k+1}=\left\{t_{0}, t_{0}, t_{1}, t_{1}, \ldots, t_{i}, t_{i}, t_{i}, \ldots, t_{p}, t_{p}\right\},
$$

where each breakpoint in $D$ has two duplicates and all the other breakpoints have only one duplicate. Construct a feasible solution $\left(\bar{v}^{k+1}, \bar{y}^{k+1}, \bar{t}^{k+1}\right)$ for $Q P\left(\left|P^{k+1}\right|\right)$ as in (18).

4. Calculate the current duality gap $d$. If the solution value of $\left(\tilde{v}^{k}, \tilde{y}^{k}, \tilde{t}^{k}\right)$ is the same as the optimal value of $A P\left(\tilde{P}^{k}\right)$, stop the algorithm. Otherwise go to Step 5.

5. Get a strictly improved solution $\left(v^{k+1}, y^{k+1}, t^{k+1}\right)$ from $\left(\bar{v}^{k+1}, \bar{y}^{k+1}, \bar{t}^{k+1}\right)$ for $Q P\left(\left|P^{k+1}\right|\right)$.

6. Let $k=k+1$.

end while

\section{Remarks.}

1. In Step 1 of Algorithm $\mathcal{A}$, we can use the Frank-Wolfe method or general matrix-splitting algorithms to compute a KKT point of $Q P\left(\left|P^{k}\right|\right)$.

2. Algorithm $\mathcal{A}$ will not loop between Step 1 and Step 2 forever, because every time Algorithm $\mathcal{A}$ goes from Step 2 to Step 1, the cardinality of $P^{k}$ is reduced at least by 1 .

3. In Step 4 of Algorithm $\mathcal{A}$, we can let $d=V\left(Q P\left(\left|\tilde{P}^{k}\right|\right)\right)-V\left(A P\left(\tilde{P}^{k}\right)\right)$. We can also let $d$ be the negative objective value of the first Frank-Wolfe iterate for $(\bar{v}, \bar{y}, \bar{t})$ and so, instead of checking whether the solution value of $\left(\tilde{v}^{k}, \tilde{y}^{k}, \tilde{t}^{k}\right)$ is the same as the optimal value of $A P\left(\tilde{P}^{k}\right)$, we can check whether the objective value of the first Frank-Wolfe iterate for $(\bar{v}, \bar{y}, \bar{t})$ is zero.

4. In Step 5 of Algorithm $\mathcal{A}$, we can use the direction constructed in section 5.3 (cf. $\left(v^{\epsilon}, y^{\epsilon}, t^{\epsilon}\right)$ ). We can also use the Frank-Wolfe method or general matrix-splitting algorithms to find a descent direction for $\left(\bar{v}^{k+1}, \bar{y}^{k+1}, \bar{t}^{k+1}\right)$. By Theorem 3, we are guaranteed to find a descent direction.

7. Convergence of the new algorithm. In this section we prove that the new algorithm converges. We first describe the argument we will use to show the convergence informally. We use the Frank-Wolfe method or general matrix-splitting algorithms to compute a series of KKT points to a series of generally nonconvex quadratic programs. These KKT points have nondecreasing solution values. By Corollary 3, we can detect whether a KKT point gives an optimal solution to $(S C S C L P)$. If it does, we terminate the algorithm. If not, by Theorem 3, we can find a new solution with approximately twice as many constant control pieces as the current solution but with a strictly improved cost. Since there is only a finite number of different solution values for the KKT points of every quadratic program constructed, and there is an upper bound on the size of the quadratic programs we encounter (see Corollary 4 below), a finite convergence result follows readily. Based on the primal solution, we can compute an optimal dual solution for $\left(S C S C L P^{*}\right)$. 
Contrary to the convergence analysis of a variety of algorithms for $(S C L P)$, we do not need to let the norm of the maximal length interval in the discretization tend to zero (as in Pullan [41]). Moreover, neither do we need the explicit knowledge of all the extreme points of a certain set of finite-dimensional linear programs (as in Anderson and Nash [2]). Most importantly, we prove the absence of a duality gap result as a byproduct of the new algorithm, even when there is no optimal solution for $(S C S C L P)$.

In the following, we give upper bounds on the cardinality of $\tilde{P}^{k}$, the purified partition in Step 2 of Algorithm $\mathcal{A}$. Since by Lemma 4 and Corollary 2 we know that the total number of zero-length intervals in $\tilde{P}^{k}$ is at most $2\left|D_{1}^{\tilde{P}^{k}}\right|$, we need only to bound the number of positive-length intervals in $\tilde{P}^{k}$. We map each positive-length interval of $\tilde{P}^{k}$ to an extreme point of a certain set of linear programs and then show that the mapping is injective. Before doing so, we give some more notation and several useful lemmas.

Let $t_{l}$ and $t_{m}$ be two consecutive breakpoints in $D_{1}^{\tilde{P}^{k}}$. By definition, $a(t), c(t)$, and $h(t)$ are linear and $b(t)$ and $g(t)$ are constant on $\left[t_{l}, t_{m}\right)$. Let $\left[\tilde{t}_{i-1}, \tilde{t}_{i}\right]$ and $\left[\tilde{t}_{i}, \tilde{t}_{i+1}\right]$ be two adjacent positive-length intervals in partition $\tilde{P}^{k}$ such that $\left[\tilde{t}_{i-1}, \tilde{t}_{i+1}\right] \subseteq\left[t_{l}, t_{m}\right]$. Let $\Delta t_{i}=\tilde{t}_{i}-\tilde{t}_{i-1}$ and $\Delta t_{i+1}=\tilde{t}_{i+1}-\tilde{t}_{i}$. We have $\Delta t_{i}>0$ and $\Delta t_{i+1}>0$ by assumption. Let $\left(\tilde{v}^{k}, \tilde{y}^{k}, \tilde{t}^{k}\right)$ be the resulting solution in Step 2 of Algorithm $\mathcal{A}$. Let $J_{i}$ be the set of indices of the constraints in $F \tilde{y}^{k}\left(\tilde{t}_{i}\right) \leq h\left(\tilde{t}_{i}\right)$ that are binding. Let

$$
\tilde{u}^{k}\left(\tilde{t}_{i-1}+\right)=\frac{\tilde{v}^{k}\left(\tilde{t}_{i}\right)}{\Delta t_{i}}
$$

and

$$
\tilde{u}^{k}\left(\tilde{t}_{i}+\right)=\frac{\tilde{v}^{k}\left(\tilde{t}_{i+1}\right)}{\Delta t_{i+1}} .
$$

It is obvious that $\left(\tilde{u}^{k}\left(\tilde{t}_{i}+\right), \frac{\tilde{y}^{k}\left(\tilde{t}_{i+1}\right)-\tilde{y}^{k}\left(\tilde{t}_{i}\right)}{\Delta t_{i+1}}\right)$ is a feasible solution to the following linear system:

$$
\begin{aligned}
\left(S Y S_{J_{i}}\right) & G \tilde{u}^{k}\left(\tilde{t}_{i}+\right)+E \frac{\tilde{y}^{k}\left(\tilde{t}_{i+1}\right)-\tilde{y}^{k}\left(\tilde{t}_{i}\right)}{\Delta t_{i+1}}=\dot{a}\left(\tilde{t}_{i}\right), \\
& H \tilde{u}^{k}\left(\tilde{t}_{i}+\right) \leq b\left(\tilde{t}_{i}\right), \\
& \left(F \frac{\tilde{y}^{k}\left(\tilde{t}_{i+1}\right)-\tilde{y}^{k}\left(\tilde{t}_{i}\right)}{\Delta t_{i+1}}\right)_{J_{i}} \leq \dot{h}\left(\tilde{t}_{i}\right), \\
& \tilde{u}^{k}\left(\tilde{t}_{i}+\right) \geq 0 .
\end{aligned}
$$

By introducing new variables, we can eliminate $\frac{\tilde{y}^{k}\left(\tilde{t}_{i+1}\right)-\tilde{y}^{k}\left(\tilde{t}_{i}\right)}{\Delta t_{i+1}}$ in $\left(S Y S_{J_{i}}\right)$ and transform $\left(S Y S_{J_{i}}\right)$ into the following linear system:

$$
\begin{aligned}
&\left(S Y S 1_{J_{i}}\right) G \tilde{u}^{k}\left(\tilde{t}_{i}+\right)+E\left(w_{i+1}-w_{i}\right)=\dot{a}\left(\tilde{t}_{i}\right), \\
& \\
& H \tilde{u}^{k}\left(\tilde{t}_{i}+\right)+\tilde{z}^{k}\left(\tilde{t}_{i}+\right)=b\left(\tilde{t}_{i}\right), \\
&\left(F\left(w_{i+1}-w_{i}\right)\right)_{J_{i}}+x=\dot{h}\left(\tilde{t}_{i}\right), \\
& x \geq 0, w_{i+1} \geq 0, w_{i} \geq 0, \tilde{u}^{k}\left(\tilde{t}_{i}+\right) \geq 0, \tilde{z}^{k}\left(\tilde{t}_{i}+\right) \geq 0 .
\end{aligned}
$$

Every extreme point of the linear program defined by maximizing some linear function over $\left(S Y S 1_{J_{i}}\right)$ defines a unique feasible solution to $\left(S Y S_{J_{i}}\right)$, which is called a generalized extreme point for $\left(S Y S_{J_{i}}\right)$. Every extreme ray of this linear program defines a 
unique ray to $\left(S Y S_{J_{i}}\right)$, which is called a generalized extreme ray for $\left(S Y S_{J_{i}}\right)$. Since this is a feasible finite-dimensional linear program in standard form, the resolution theorem applies. After translating the result into variables in $\left(S Y S_{J_{i}}\right)$, we have the following analogue of the resolution theorem for $\left(S Y S_{J_{i}}\right)$.

LEMMA 5. Every feasible solution of $\left(S Y S_{J_{i}}\right)$ can be written as the sum of a convex combination of the generalized extreme points of $\left(S Y S_{J_{i}}\right)$ and a linear combination (with nonnegative coefficients) of generalized extreme rays to $\left(S Y S_{J_{i}}\right)$.

By Lemma 5, we have

$$
\begin{aligned}
\tilde{u}^{k}\left(\tilde{t}_{i}+\right) & =\sum_{j=1}^{k^{(i)}} \lambda_{j}^{(i)} s_{j}^{(i)}+\sum_{j=1}^{q^{(i)}} \mu_{j}^{(i)} r_{j}^{(i)} \\
\frac{\tilde{y}^{k}\left(\tilde{t}_{i+1}\right)-\tilde{y}^{k}\left(\tilde{t}_{i}\right)}{\Delta t_{i+1}} & =\sum_{j=1}^{k^{(i)}} \lambda_{j}^{(i)} \bar{s}_{j}^{(i)}+\sum_{j=1}^{q^{(i)}} \mu_{j}^{(i)} \bar{r}_{j}^{(i)}
\end{aligned}
$$

for some positive $k^{(i)} \geq 1$ and nonnegative $q^{(i)} \geq 0$, where $\lambda_{j}^{(i)}>0, \sum_{j=1}^{k^{(i)}} \lambda_{j}^{(i)}=1$, and $\mu_{j}^{(i)}>0$, the $\left(s_{j}^{(i)}, \bar{s}_{j}^{(i)}\right)$ are generalized extreme points to system $\left(S Y S_{J_{i}}\right)$, and the $\left(r_{j}^{(i)}, \bar{r}_{j}^{(i)}\right)$ are generalized extreme rays to system $\left(S Y S_{J_{i}}\right)$. Without loss of generality, assume that we have sorted $\left(s_{j}^{(i)}, \bar{s}_{j}^{(i)}\right)$ in the following order:

$$
\dot{c}\left(\tilde{t}_{i}\right)^{\prime} s_{j}^{(i)}-g\left(t_{l}+\right)^{\prime} \bar{s}_{j}^{(i)} \geq \dot{c}\left(\tilde{t}_{i}\right)^{\prime} s_{j+1}^{(i)}-g\left(t_{l}+\right)^{\prime} \bar{s}_{j+1}^{(i)} \quad \text { for all } j .
$$

We have the following result on $\left(\tilde{v}^{k}, \tilde{y}^{k}, \tilde{t}^{k}\right)$.

LEMMA 6.

$$
V\left(Q P\left(\left|\tilde{P}^{k}\right|\right),\left(\tilde{v}^{k}, \tilde{y}^{k}, \tilde{t}^{k}\right)\right) \leq V\left(Q P\left(\left|P^{k}\right|\right),\left(v^{k}, y^{k}, t^{k}\right)\right) .
$$

Proof. Since Procedure PURIFY does not increase the solution value of the current solution, the result immediately follows.

Lemma 7. Suppose (30) and (31) hold for $\tilde{u}^{k}\left(\tilde{t}_{i-1}+\right)$ and $\tilde{u}^{k}\left(\tilde{t}_{i}+\right)$. Furthermore, suppose $\left[\tilde{t}_{i-1}, \tilde{t}_{i}\right]$ is not the first positive-length interval that resides on $\left[t_{l}, t_{m}\right]$. Then we have

$$
\dot{c}\left(\tilde{t}_{i}\right)^{\prime} s_{1}^{(i-1)}-g\left(t_{l}+\right)^{\prime} \bar{s}_{1}^{(i-1)}>\dot{c}\left(\tilde{t}_{i}\right)^{\prime} s_{1}^{(i)}-g\left(t_{l}+\right)^{\prime} \bar{s}_{1}^{(i)}
$$

for the two adjacent positive-length intervals $\left[\tilde{t}_{i-1}, \tilde{t}_{i}\right]$ and $\left[\tilde{t}_{i}, \tilde{t}_{i+1}\right]$ that reside on $\left[t_{l}, t_{m}\right]$.

Proof. We first show that

$$
\dot{c}\left(\tilde{t}_{i}\right)^{\prime} r_{j}^{(i)}-g\left(t_{l}+\right)^{\prime} \bar{r}_{j}^{(i)} \leq 0
$$

for every $j \leq q^{(i)}$ without assuming that $\left[\tilde{t}_{i-1}, \tilde{t}_{i}\right]$ is not the first positive-length interval that resides on $\left[t_{l}, t_{m}\right]$.

Let $\tau \in(0,1)$. Suppose

$$
\tilde{u}^{k}\left(\tilde{t}_{i}+\right)=\tau u_{1}+(1-\tau) u_{2}
$$

and

$$
\frac{\tilde{y}^{k}\left(\tilde{t}_{i+1}\right)-\tilde{y}^{k}\left(\tilde{t}_{i}\right)}{\Delta t_{i+1}}=\tau y_{1}+(1-\tau) y_{2},
$$




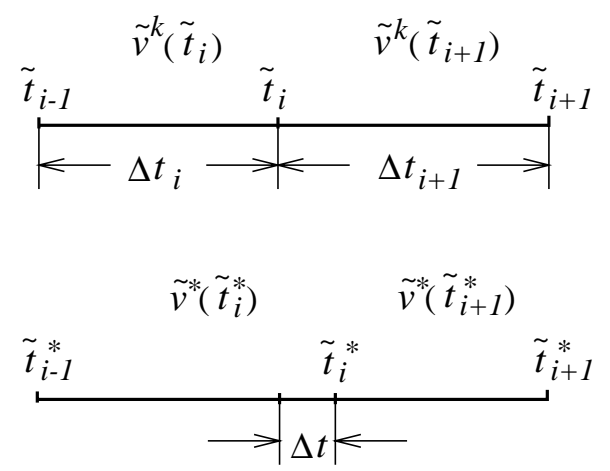

FIG. 3. Perturbation of the solution.

where $\left(u_{1}, y_{1}\right)$ and $\left(u_{2}, y_{2}\right)$ are two feasible solutions for $\left(S Y S_{J_{i}}\right)$. Let $\gamma$ be the largest scalar in $\left(0, \tau \Delta t_{i+1}\right]$ such that $F\left(\tilde{y}^{k}\left(\tilde{t}_{i}\right)+\gamma y_{1}\right) \leq h\left(\tilde{t}_{i}\right)$. Such a $\gamma$ exists by virtue of the feasibility of $\left(u_{1}, y_{1}\right)$ and $\left(u_{2}, y_{2}\right)$ to system $\left(S Y S_{J_{i}}\right)$. For any $\Delta t \in(0, \gamma)$, we consider the following perturbation of $\left(\tilde{v}^{k}, \tilde{y}^{k}, \tilde{t}^{k}\right)$, as shown in Figure 3 :

$$
\begin{aligned}
\tilde{t}_{j}^{*} & = \begin{cases}\tilde{t}_{i}+\Delta t & \text { if } j=i, \\
\tilde{t}_{j} & \text { otherwise, }\end{cases} \\
\tilde{v}^{*}\left(\tilde{t}_{j}^{*}\right) & = \begin{cases}\tilde{v}^{k}\left(\tilde{t}_{i}\right)+u_{1} \Delta t & \text { if } j=i, \\
\tilde{v}^{k}\left(\tilde{t}_{j}\right)-u_{1} \Delta t & \text { if } j=i+1, \\
\tilde{v}^{k}\left(\tilde{t}_{j}\right) & \text { otherwise, }\end{cases} \\
\tilde{y}^{*}\left(\tilde{t}_{j}^{*}\right) & = \begin{cases}\tilde{y}^{k}\left(\tilde{t}_{i}\right)+y_{1} \Delta t & \text { if } j=i, \\
\tilde{y}^{k}\left(\tilde{t}_{j}\right) & \text { otherwise. }\end{cases}
\end{aligned}
$$

We can easily check the feasibility of $\left(\tilde{v}^{*}, \tilde{y}^{*}, \tilde{t}^{*}\right)$ to $Q P(|\tilde{P}|)$. So

$$
\begin{aligned}
& \sum_{j=1}^{p} \tilde{v}^{*}\left(\tilde{t}_{j}^{*}\right)^{\prime} c\left(\frac{\tilde{t}_{i-1}^{*}+\tilde{t}_{i}^{*}}{2}\right)-\sum_{j=1}^{p} \tilde{v}^{k}\left(\tilde{t}_{j}\right)^{\prime} c\left(\frac{\tilde{t}_{i-1}+\tilde{t}_{i}}{2}\right) \\
= & \left(\frac{c\left(\tilde{t}_{i-1}\right)+c\left(\tilde{t}_{i}^{*}\right)}{2}\right)^{\prime} \tilde{v}^{*}\left(\tilde{t}_{i}^{*}\right)+\left(\frac{c\left(\tilde{t}_{i}^{*}\right)+c\left(\tilde{t}_{i+1}\right)}{2}\right)^{\prime} \tilde{v}^{*}\left(\tilde{t}_{i+1}^{*}\right) \\
& -\left(\frac{c\left(\tilde{t}_{i-1}\right)+c\left(\tilde{t}_{i}\right)}{2}\right)^{\prime} \tilde{v}^{k}\left(\tilde{t}_{i}\right)-\left(\frac{c\left(\tilde{t}_{i}\right)+c\left(\tilde{t}_{i+1}\right)}{2}\right)^{\prime} \tilde{v}^{k}\left(\tilde{t}_{i+1}\right) \\
= & \left(\frac{c\left(\tilde{t}_{i}^{*}\right)-c\left(\tilde{t}_{i}\right)}{2}\right)^{\prime} \tilde{v}^{k}\left(\tilde{t}_{i}\right)+\left(\frac{c\left(\tilde{t}_{i}^{*}\right)-c\left(\tilde{t}_{i}\right)}{2}\right)^{\prime} \tilde{v}^{k}\left(\tilde{t}_{i+1}\right) \\
& -\frac{\Delta t}{2}\left(\Delta t_{i}+\Delta t_{i+1}\right) \dot{c}\left(\tilde{t}_{i}\right)^{\prime} u_{1} \\
= & \frac{\Delta t \Delta t_{i}}{2}\left(\dot{c}\left(\tilde{t}_{i}\right)^{\prime} \tilde{u}^{k}\left(\tilde{t}_{i}-\right)-\dot{c}\left(\tilde{t}_{i}\right)^{\prime} u_{1}\right) \\
& +\frac{\Delta t \Delta t_{i+1}}{2}\left(\dot{c}\left(\tilde{t}_{i}\right)^{\prime} \tilde{u}^{k}\left(\tilde{t}_{i}+\right)-\dot{c}\left(\tilde{t}_{i}\right)^{\prime} u_{1}\right) .
\end{aligned}
$$

Also,

$$
\sum_{j=1}^{p} \frac{\tilde{t}_{i}^{*}-\tilde{t}_{i-1}^{*}}{2}\left(\tilde{y}^{*}\left(\tilde{t}_{i}^{*}\right)+\tilde{y}^{*}\left(\tilde{t}_{i-1}^{*}\right)\right)^{\prime} g\left(\tilde{t}_{i-1}^{*}+\right)
$$




$$
\begin{aligned}
& -\sum_{j=1}^{p} \frac{\tilde{t}_{i}-\tilde{t}_{i-1}}{2}\left(\tilde{y}^{k}\left(\tilde{t}_{i}\right)+\tilde{y}^{k}\left(\tilde{t}_{i-1}\right)\right)^{\prime} g\left(\tilde{t}_{i-1}+\right) \\
= & \frac{\tilde{t}_{i}^{*}-\tilde{t}_{i-1}^{*}}{2}\left(\tilde{y}^{*}\left(\tilde{t}_{i}^{*}\right)+\tilde{y}^{*}\left(\tilde{t}_{i-1}^{*}\right)\right)^{\prime} g\left(\tilde{t}_{i-1}^{*}+\right) \\
& +\frac{\tilde{t}_{i+1}^{*}-\tilde{t}_{i}^{*}}{2}\left(\tilde{y}^{*}\left(\tilde{t}_{i+1}^{*}\right)+\tilde{y}^{*}\left(\tilde{t}_{i}^{*}\right)\right)^{\prime} g\left(\tilde{t}_{i}^{*}+\right) \\
& -\frac{\tilde{t}_{i}-\tilde{t}_{i-1}}{2}\left(\tilde{y}^{k}\left(\tilde{t}_{i}\right)+\tilde{y}^{k}\left(\tilde{t}_{i-1}\right)\right)^{\prime} g\left(\tilde{t}_{i-1}+\right) \\
& -\frac{\tilde{t}_{i+1}-\tilde{t}_{i}}{2}\left(\tilde{y}^{k}\left(\tilde{t}_{i+1}\right)+\tilde{y}^{k}\left(\tilde{t}_{i}\right)\right)^{\prime} g\left(\tilde{t}_{i}+\right) \\
= & \frac{\Delta t_{i}+\Delta t}{2}\left(\tilde{y}^{k}\left(\tilde{t}_{i}\right)+\tilde{y}^{k}\left(\tilde{t}_{i-1}\right)+\Delta t y_{1}\right)^{\prime} g\left(\tilde{t}_{i-1}+\right) \\
& +\frac{\Delta t_{i+1}-\Delta t}{2}\left(\tilde{y}^{k}\left(\tilde{t}_{i+1}\right)+\tilde{y}^{k}\left(\tilde{t}_{i}\right)+\Delta t y_{1}\right)^{\prime} g\left(\tilde{t}_{i}+\right) \\
& -\frac{\Delta t_{i}}{2}\left(\tilde{y}^{k}\left(\tilde{t}_{i}\right)+\tilde{y}^{k}\left(\tilde{t}_{i-1}\right)\right)^{\prime} g\left(\tilde{t}_{i-1}+\right)-\frac{\Delta t_{i+1}}{2}\left(\tilde{y}^{k}\left(\tilde{t}_{i+1}\right)+\tilde{y}^{k}\left(\tilde{t}_{i}\right)\right)^{\prime} g\left(\tilde{t}_{i}+\right) \\
= & \frac{\Delta t}{2}\left(\tilde{y}^{k}\left(\tilde{t}_{i-1}\right)-\tilde{y}^{k}\left(\tilde{t}_{i+1}\right)+\left(\Delta t_{i}+\Delta t_{i+1}\right) y_{1}\right)^{\prime} g\left(t_{l}+\right) .
\end{aligned}
$$

Combining (33) and (34), we derive

$$
\begin{aligned}
& V\left(Q P\left(\left|\tilde{P}^{k}\right|\right),\left(\tilde{v}^{*}, \tilde{y}^{*}, \tilde{t}^{*}\right)\right)-V\left(Q P\left(\left|\tilde{P}^{k}\right|\right),\left(\tilde{v}^{k}, \tilde{y}^{k}, \tilde{t}^{k}\right)\right) \\
= & \frac{\Delta t \Delta t_{i}}{2}\left(\dot{c}\left(\tilde{t}_{i}\right)^{\prime} \tilde{u}^{k}\left(\tilde{t}_{i}-\right)-g\left(\tilde{t}_{i}+\right)^{\prime} \frac{\tilde{y}^{k}\left(\tilde{t}_{i}\right)-\tilde{y}^{k}\left(\tilde{t}_{i-1}\right)}{\Delta t_{i}}-\left(\dot{c}\left(\tilde{t}_{i}\right)^{\prime} u_{1}-y_{1}^{\prime} g\left(t_{l}+\right)\right)\right) \\
& +\frac{\Delta t \Delta t_{i+1}}{2}\left(\dot{c}\left(\tilde{t}_{i}\right)^{\prime} \tilde{u}^{k}\left(\tilde{t}_{i}+\right)-g\left(\tilde{t}_{i}+\right)^{\prime} \frac{\tilde{y}^{k}\left(\tilde{t}_{i+1}\right)-\tilde{y}^{k}\left(\tilde{t}_{i}\right)}{\Delta t_{i+1}}-\left(\dot{c}\left(\tilde{t}_{i}\right)^{\prime} u_{1}-y_{1}^{\prime} g\left(t_{l}+\right)\right)\right) .
\end{aligned}
$$

By the definition of a KKT point and the discussion following it in section 3.1, a feasible solution to $Q P(|P|)$ is a KKT point if and only if there is no feasible descent direction for this solution. Hence

$$
V\left(Q P\left(\left|\tilde{P}^{k}\right|\right),\left(\tilde{v}^{*}, \tilde{y}^{*}, \tilde{t}^{*}\right)\right)-V\left(Q P\left(\left|\tilde{P}^{k}\right|\right),\left(\tilde{v}^{k}, \tilde{y}^{k}, \tilde{t}^{k}\right)\right) \geq 0 .
$$

Thus (35) implies that $\dot{c}\left(\tilde{t}_{i}\right)^{\prime} u_{1}-y_{1}^{\prime} g\left(t_{l}+\right)$ is uniformly bounded from above for any possible choice of $\left(u_{1}, y_{1}\right)$.

For any $\bar{j} \leq q^{(i)}$ and any $\epsilon \in(0,1)$, we have

$$
\begin{aligned}
\tilde{u}^{k}\left(\tilde{t}_{i}+\right)= & \epsilon \lambda_{1}^{(i)}\left(s_{1}^{(i)}+\frac{\mu_{\bar{j}}^{(i)}}{\epsilon \lambda_{1}^{(i)}} r_{\bar{j}}^{(i)}\right) \\
& +\left(1-\epsilon \lambda_{1}^{(i)}\right)\left(\sum_{j=2}^{k^{(i)}} \frac{\lambda_{j}^{(i)}}{1-\epsilon \lambda_{1}^{(i)}} s_{j}^{(i)}+\frac{\lambda_{1}^{(i)}(1-\epsilon)}{1-\epsilon \lambda_{1}^{(i)}} s_{1}^{(1)}+\sum_{j=1, j \neq \bar{j}}^{q^{(i)}} \frac{\mu_{j}^{(i)}}{1-\epsilon \lambda_{1}^{(i)}} r_{j}^{(i)}\right)
\end{aligned}
$$

and

$$
\frac{\tilde{y}^{k}\left(\tilde{t}_{i+1}\right)-\tilde{y}^{k}\left(\tilde{t}_{i}\right)}{\Delta t_{i+1}}
$$




$$
\begin{aligned}
= & \epsilon \lambda_{1}^{(i)}\left(\bar{s}_{1}^{(i)}+\frac{\mu_{\bar{j}}^{(i)}}{\epsilon \lambda_{1}^{(i)}} \bar{r}_{\bar{j}}^{(i)}\right) \\
& +\left(1-\epsilon \lambda_{1}^{(i)}\right)\left(\sum_{j=2}^{k^{(i)}} \frac{\lambda_{j}^{(i)}}{1-\epsilon \lambda_{1}^{(i)}} \bar{s}_{j}^{(i)}+\frac{\lambda_{1}^{(i)}(1-\epsilon)}{1-\epsilon \lambda_{1}^{(i)}} \bar{s}_{1}^{(1)}+\sum_{j=1, j \neq j}^{q^{(i)}} \frac{\mu_{j}^{(i)}}{1-\epsilon \lambda_{1}^{(i)}} \bar{r}_{j}^{(i)}\right) .
\end{aligned}
$$

By letting

$$
u_{1}=s_{1}^{(i)}+\frac{\mu_{\bar{j}}^{(i)}}{\epsilon \lambda_{1}^{(i)}} r_{\bar{j}}^{(i)}, \quad y_{1}=\bar{s}_{1}^{(i)}+\frac{\mu_{\bar{j}}^{(i)}}{\epsilon \lambda_{1}^{(i)}} \bar{r}_{\bar{j}}^{(i)},
$$

and letting $\epsilon$ tend to zero, the above boundedness result on $\dot{c}\left(\tilde{t}_{i}\right)^{\prime} u_{1}^{(i)}-y_{1}^{\prime} g\left(t_{l}+\right)$ implies (32). Since $\left[t_{i-1}, t_{i}\right]$ is not the first positive-length interval that resides on $\left[t_{l}, t_{m}\right]$, we can similarly have

$$
\dot{c}\left(\tilde{t}_{i}\right)^{\prime} r_{j}^{(i-1)}-g\left(t_{l}+\right)^{\prime} \bar{r}_{j}^{(i-1)} \leq 0 \text { for all } j .
$$

These together with (30) and (31) give

$$
\dot{c}\left(\tilde{t}_{i}\right)^{\prime} s_{1}^{(i-1)}-g\left(t_{l}+\right)^{\prime} \bar{s}_{1}^{(i-1)} \geq \dot{c}\left(\tilde{t}_{i}\right)^{\prime} \tilde{u}^{k}\left(\tilde{t}_{i-1}+\right)-g\left(\tilde{t}_{i}+\right)^{\prime} \frac{\tilde{y}^{k}\left(\tilde{t}_{i}\right)-\tilde{y}^{k}\left(\tilde{t}_{i-1}\right)}{\Delta t_{i}} .
$$

Similarly,

$$
\dot{c}\left(\tilde{t}_{i}\right)^{\prime} s_{1}^{(i)}-g\left(t_{l}+\right)^{\prime} \bar{s}_{1}^{(i)} \geq \dot{c}\left(\tilde{t}_{i}\right)^{\prime} \tilde{u}^{k}\left(\tilde{t}_{i}+\right)-g\left(\tilde{t}_{i}+\right)^{\prime} \frac{\tilde{y}^{k}\left(\tilde{t}_{i+1}\right)-\tilde{y}^{k}\left(\tilde{t}_{i}\right)}{\Delta t_{i+1}} .
$$

Since $\tilde{P}$ is a purified partition, by Procedure PURIFY, the opposite of (11) holds, which is equivalent to

$\dot{c}\left(\tilde{t}_{i}\right)^{\prime} \tilde{u}^{k}\left(\tilde{t}_{i-1}+\right)-g\left(\tilde{t}_{i}+\right)^{\prime} \frac{\tilde{y}^{k}\left(\tilde{t}_{i}\right)-\tilde{y}^{k}\left(\tilde{t}_{i-1}\right)}{\Delta t_{i}}>\dot{c}\left(\tilde{t}_{i}\right)^{\prime} \tilde{u}^{k}\left(\tilde{t}_{i}+\right)-g\left(\tilde{t}_{i}+\right)^{\prime} \frac{\tilde{y}^{k}\left(\tilde{t}_{i+1}\right)-\tilde{y}^{k}\left(\tilde{t}_{i}\right)}{\Delta t_{i+1}}$.

Now, suppose

$$
\dot{c}\left(\tilde{t}_{i}\right)^{\prime} s_{1}^{(i-1)}-g\left(t_{l}+\right)^{\prime} \bar{s}_{1}^{(i-1)} \leq \dot{c}\left(\tilde{t}_{i}\right)^{\prime} s_{1}^{(i)}-g\left(t_{l}+\right)^{\prime} \bar{s}_{1}^{(i)} .
$$

By (37) and (39), we have

$$
\dot{c}\left(\tilde{t}_{i}\right)^{\prime} \tilde{u}^{k}\left(\tilde{t}_{i}+\right)-g\left(\tilde{t}_{i}+\right)^{\prime} \frac{\tilde{y}^{k}\left(\tilde{t}_{i+1}\right)-\tilde{y}^{k}\left(\tilde{t}_{i}\right)}{\Delta t_{i+1}}<\dot{c}\left(\tilde{t}_{i}\right)^{\prime} s_{1}^{(i)}-g\left(t_{l}+\right)^{\prime} \bar{s}_{1}^{(i)}
$$

and

$$
\dot{c}\left(\tilde{t}_{i}\right)^{\prime} \tilde{u}^{k}\left(\tilde{t}_{i-1}+\right)-g\left(\tilde{t}_{i}+\right)^{\prime} \frac{\tilde{y}^{k}\left(\tilde{t}_{i}\right)-\tilde{y}^{k}\left(\tilde{t}_{i-1}\right)}{\Delta t_{i}} \leq \dot{c}\left(\tilde{t}_{i}\right)^{\prime} s_{1}^{(i)}-g\left(t_{l}+\right)^{\prime} \bar{s}_{1}^{(i)}
$$

Let $u_{1}=s_{1}^{(i)}$ and $y_{1}=\bar{s}_{1}^{(i)}$. Then the above relationship together with (35) gives

$$
V\left(Q P\left(\left|\tilde{P}^{k}\right|\right),\left(\tilde{v}^{*}, \tilde{y}^{*}, \tilde{t}^{*}\right)\right)-V\left(Q P\left(\left|\tilde{P}^{k}\right|\right),\left(\tilde{v}^{k}, \tilde{y}^{k}, \tilde{t}^{k}\right)\right)<0,
$$

which contradicts that $\left(\tilde{v}^{k}, \tilde{y}^{k}, \tilde{t}^{k}\right)$ is a KKT point for $Q P\left(\left|\tilde{P}^{k}\right|\right)$ (cf. (36)). 
Since $\dot{c}\left(\tilde{t}_{i}\right)$ is a constant vector over $\left[t_{l}, t_{m}\right]$, as a consequence of Lemma 7 , every nonzero-length interval that resides on $\left[t_{l}, t_{m}\right]$ (except the first nonzero-length interval) corresponds to a different generalized extreme point of some system $\left(S Y S_{J_{i}}\right)$. Since only a finite number of different systems $\left(S Y S_{J_{i}}\right)$ exists, and for each $\left(S Y S_{J_{i}}\right)$ there is a finite number of generalized extreme points, we see there is only a finite number of nonzero-length intervals that reside on $\left[t_{l}, t_{m}\right]$. Since the number of zerolength intervals that reside on $\left[t_{l}, t_{m}\right]$ is at most two (one on each end of $\left[t_{l}, t_{m}\right]$ ), there is also a finite number of breakpoints in $\left[t_{l}, t_{m}\right]$. Thus we have the following corollary.

COROLlary 4. There is a finite number of breakpoints in $\tilde{P}^{k}$.

There is only a finite number of different solution values for all the KKT points of $Q P(|P|)$, as shown in the following lemma.

LEMMA 8. The KKT points for $Q P(|P|)$ are the union of a finite number of connected sets. Over each connected component of KKT points of $Q P(|P|)$, the objective value is a constant. Furthermore, the number of connected sets is bounded from above by a number that depends on $|P|$ only.

Proof. It is easily seen that a solution to $Q P(|P|)$ is a KKT point of $Q P(|P|)$ if and only if it is a solution to a feasible symmetric affine variational inequality problem whose dimension depends only on $|P|$ (cf. section 3.1). The lemma now follows directly from Lemma 3.1 of Luo and Tseng [31].

We now present the main convergence result of the paper.

TheOREM 5. Algorithm $\mathcal{A}$ will terminate after a finite number of iterations.

Proof. Suppose Algorithm $\mathcal{A}$ does not terminate after a finite number of iterations. It is guaranteed by Theorem 3 that Step 4 of Algorithm $\mathcal{A}$ would produce a strictly improved solution, and thus every iteration of Algorithm $\mathcal{A}$ would give a KKT point of a certain $Q P(|P|)$ that has a strictly better solution value. By Lemma 8 , the KKT points generated by $Q P(|P|)$ should lie on a different connected KKT points component of $Q P(|P|)$ for every $|P|$. This means that the cardinality of $P$ is unbounded and contradicts Corollary 4.

8. New structural and duality results. As a result of Algorithm $\mathcal{A}$ and Theorem 5, we have the following new structural result for (SCSCLP).

Theorem 6. Under Assumption 1, Algorithm $\mathcal{A}$ terminates with a solution to $Q P(|P|)$ for some $P$ that gives the optimal objective value of (SCSCLP) and can be closely approximated by a series of piecewise constant controls for (SCSCLP). When the solution set for (SCSCLP) is bounded and $E$ is an identity matrix, Algorithm $\mathcal{A}$ terminates with a piecewise constant optimal control with partition $P$ such that $t_{i} \neq t_{i-1}$ for all $i$. Furthermore, over each interval $\left[t_{i-1}, t_{i}\right),\left(u\left(t_{i}+\right), \frac{y\left(t_{i+1}\right)-y\left(t_{i}\right)}{t_{i+1}-t_{i}}\right)$ is a convex combination of the generalized extreme points of linear system $\left(S Y S_{J_{i}}\right)$, where $J_{i}$ is a subset of $\left\{1, \ldots, n_{2}\right\}$.

Proof. The first part of the theorem is a direct consequence of Theorem 5. The second part of the theorem follows from Lemma 5 and the remark following the proof of Lemma 1.

We remark that when the solution set for $(S C S C L P)$ is unbounded, it is possible that the optimal solution value is not attained. We next derive the following new duality result for $(S C S C L P)$.

THEOREM 7. Under Assumption 1, there is no duality gap between (SCSCLP) and $\left(S C S C L P^{*}\right)$. There always exists an optimal solution for $\left(S C S C L P^{*}\right)$ that is piecewise linear. Furthermore, there exists a bounded measurable optimal solution for $(S C S C L P)$ if and only if Algorithm $\mathcal{A}$ terminates with such a solution. 
Proof. The first part of the theorem is a direct consequence of Theorem 5 .

Denote $\tilde{P}^{k}$ as the final purified partition when Algorithm $\mathcal{A}$ terminates. To prove the second part of the theorem, we first show that the zero-length intervals in $\tilde{P}^{k}$ can be eliminated in the dual problem $A P^{*}(P)$. Let $\left[t_{i-1}, t_{i}\right]$ be a zero-length interval that resides on $\left[t_{l}, t_{m}\right]$, where $t_{l}$ and $t_{m}$ are two consecutive breakpoints in $D_{1}^{\tilde{P}^{k}}$. By Lemma 4 , the zero-length intervals can be located only at the breakpoints in $D_{1}^{\tilde{P}^{k}}$. We assume $t_{i}=t_{m}$ (the case $t_{i-1}=t_{l}$ can be treated similarly).

Let $(\hat{\pi}, \hat{\eta}, \hat{\xi})$ be an optimal solution for $A P^{*}\left(\tilde{P}^{k}\right)$. Then we can construct a new solution $(\tilde{\pi}, \tilde{\eta}, \tilde{\xi})$ for $A P^{*}\left(\tilde{P}^{k}\right)$ in the following way. Let $(\tilde{\pi}, \tilde{\eta}, \tilde{\xi})$ equal $(\hat{\pi}, \hat{\eta}, \hat{\xi})$ except

$$
\begin{aligned}
\tilde{\pi}\left(t_{i-1}+\right) & =\hat{\pi}\left(t_{i-1}-\right), \quad \tilde{\pi}\left(t_{i}-\right)=\hat{\pi}\left(t_{i-1}-\right), \\
\tilde{\eta}\left(t_{i-1}+\right) & =\hat{\eta}\left(t_{i-1}-\right), \quad \tilde{\eta}\left(t_{i}-\right)=\hat{\eta}\left(t_{i-1}-\right), \\
\tilde{\xi}\left(\frac{t_{i-1}+t_{i}}{2}\right) & =0, \quad \tilde{\xi}\left(t_{i-1}\right)=0, \\
\tilde{\xi}\left(t_{i}\right) & =\hat{\xi}\left(\frac{t_{i-1}+t_{i}}{2}\right)+\hat{\xi}\left(t_{i-1}\right)+\hat{\xi}\left(t_{i}\right) .
\end{aligned}
$$

It is easy to check the feasibility of $(\tilde{\pi}, \tilde{\eta}, \tilde{\xi})$. It is a fact that $(\tilde{\pi}, \tilde{\eta}, \tilde{\xi})$ and $(\hat{\pi}, \hat{\eta}, \hat{\xi})$ have the same solution value in $A P^{*}\left(\tilde{P}^{k}\right)$. Let $\bar{P}$ be $\tilde{P}^{k} \backslash\left\{t_{i-1}\right\}$. By eliminating the elements $\tilde{\pi}\left(t_{i-1}-\right), \tilde{\pi}\left(t_{i-1}+\right), \tilde{\eta}\left(t_{i-1}-\right), \tilde{\eta}\left(t_{i-1}+\right), \tilde{\xi}\left(t_{i-1}\right)$, and $\tilde{\xi}\left(\frac{t_{i-1}+t_{i}}{2}\right)$ from $(\tilde{\pi}, \tilde{\eta}, \tilde{\xi})$, we can get a feasible solution $(\bar{\pi}, \bar{\eta}, \bar{\xi})$ for $A P(\bar{P})$. Also, $(\bar{\pi}, \bar{\eta}, \bar{\xi})$ has the same solution value as $(\tilde{\pi}, \tilde{\eta}, \tilde{\xi})$.

By repeating this process, we can eliminate all the zero-length intervals in $\tilde{P}^{k}$ and define a feasible solution for $A P^{*}(P)$ from the resulting partition $P$. From this feasible solution, we can construct an optimal solution for $\left(S C S C L P^{*}\right)$ that is piecewise linear. This proves the second part of the theorem.

One direction of the third part of the theorem is quite obvious. The other direction (i.e., if there exists a bounded measurable optimal solution for (SCSCLP), then Algorithm $\mathcal{A}$ will find such a solution) can be shown as follows. Let the bounded measurable solution $(u(t), y(t))$ be optimal for $(S C S C L P)$. By the second part of the theorem, there always exists an optimal solution $(\pi(t), \eta(t), \xi(t))$ for $\left(S C S C L P^{*}\right)$ that is piecewise linear with partition $P$ (defined by removing all the zero-length intervals from $\left.\tilde{P}^{K}\right)$. By Corollary 1 , the complementary slackness condition (5) is satisfied. Let $\bar{u}(t)$ be the piecewise constant extensions of $u\left(t_{0}+\right), u\left(t_{1}+\right), \ldots, u\left(t_{p-1}+\right)$. Let $\bar{y}(t)$ be the piecewise-linear extension of $y\left(t_{0}+\right), y\left(t_{1}-\right) y\left(t_{1}+\right), \ldots, y\left(t_{p-1}+\right), y\left(t_{p}-\right)$. The solution $(\bar{u}(t), \bar{y}(t))$ is a feasible solution for $(S C S C L P)$ which together with $(\pi(t), \eta(t), \xi(t))$ satisfies $(5)$. Therefore, Corollary 1 again, $(\bar{u}(t), \bar{y}(t))$ is optimal for $(S C S C L P)$.

9. Computational results. Algorithm $\mathcal{A}$ has been implemented and tested on a Sparc 10/41. The program is written in $C$. We used the academic version of $L O Q O$ Version 1.08 by Vanderbei [48]. We call its subroutines to solve intermediate linear programming and quadratic programming subproblems.

The implementation of Algorithm $\mathcal{A}$ consists of four modules: the input data processing module, the output module, the successive quadratic programming module, and the lower bound module. The successive quadratic programming module uses the Frank-Wolfe method to iteratively solve a sequence of quadratic programs, as outlined in Algorithm $\mathcal{A}$. The lower bound module uses the partition generated by 


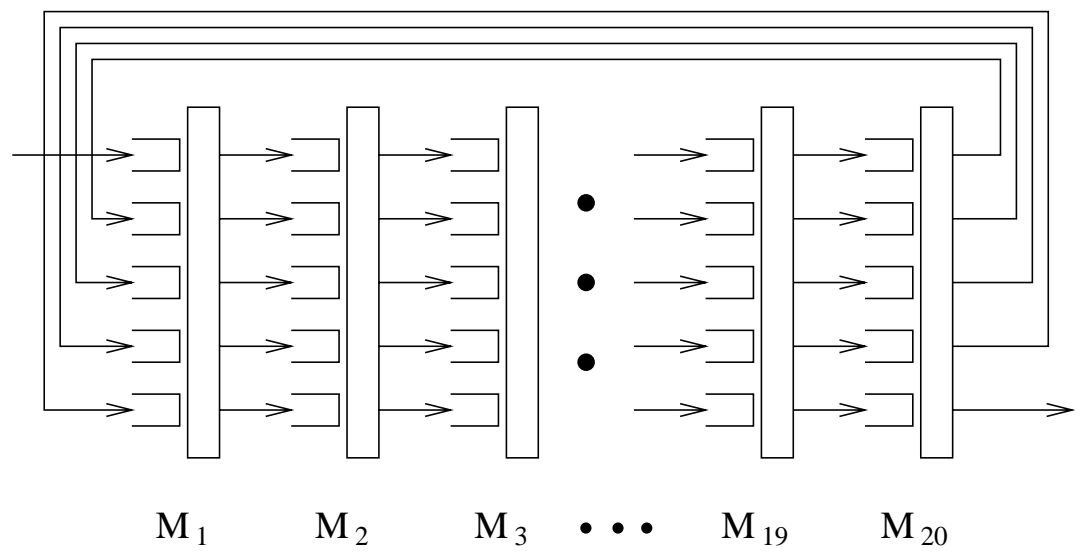

FIG. 4. The reentrant line for the example.

the successive quadratic programming module to calculate a dual feasible solution for the problem.

We next give a numerical example that arises in manufacturing systems. The example is a reentrant line, as in Kumar [25]. A reentrant line is a multiclass queuing network with fixed routing.

The reentrant line we consider is shown in Figure 4. We have from left to right $n$ stations (in Figure 4, we have 20 stations), and each station services 5 different classes of customers. There are $5 n$ classes of customers in total. Class $i$ customers will be served at machine $\lfloor(i-1) / n+1\rfloor$. After class $i$ customer finishes service, it will become class $i+1$ customer if $i<5 n$ and exit the system otherwise. For this system, we assume the exogenous arrival rate for class 1 customer is 1 and is zero for all other classes. We generate randomly the mean service time, the cost per unit time, and the initial number of customers for each class of customers. Our objective is to find an optimal control policy (involving both routing and sequencing decisions) that minimizes the cumulated cost of queuing over a fixed time horizon $[0, T]$.

We can formulate the problem as an $(S C S C L P)$. Let $y_{i}(t)$ be the queue length of class $i$ customers at time $t$. If class $i$ customers are served at machine $j$, we let $u_{i}(t)$ be the proportion of machine capacity of machine $j$ that is devoted to class $i$ customers at time $t$. The $G$ matrix of the $(S C S C L P)$ is the node-arc incidence matrix for the following line digraph: Node $i$ of the graph corresponds to class $i$ and the edges are $(i, i+1)$ for $i=1, \ldots, 5 n-1$. The matrix $H$ is a block diagonal matrix, with each block a row vector of mean service times of the customers served at the same machine. $F$ is a negative identity matrix. $c(t)$ is a zero vector. $g(t)$ is a randomly generated vector. $a(t)=y(0)+e_{1} t$ with $e_{1}$ the unit vector whose first component is one and all the other components are zero. $b(t)$ is a vector of all ones and $h(t)$ is a zero vector.

The computational sequences are shown in Table 9.1.

When we fix the precision requirement and vary the number of stations in the example, we find that the computational time grows almost quadratically with the problem dimension, as shown in Figure 5. This is due to the fact that the number of control pieces grows almost linearly with the problem dimension and the total number of nonzero elements in the intermediate problems grows almost quadratically with the number of stations. Notice that for the largest example in Figure 5 (25 stations) there are 250 continuous variables. 
TABLE 9.1

Test results for the example.

\begin{tabular}{|c|c|c|c|c|}
\hline \# Iter. & Obj. Value & \# Pieces & Dual obj. & Time in sec. \\
\hline \hline 0 & 20987.1355 & 7 & & \\
\hline 1 & 5986.7656 & 7 & 5956.7923 & 134.05 \\
\hline 2 & 5965.1006 & 15 & 5962.7291 & 1738.2 \\
\hline 3 & 5963.6674 & 29 & 5963.2700 & 2436.61 \\
\hline
\end{tabular}

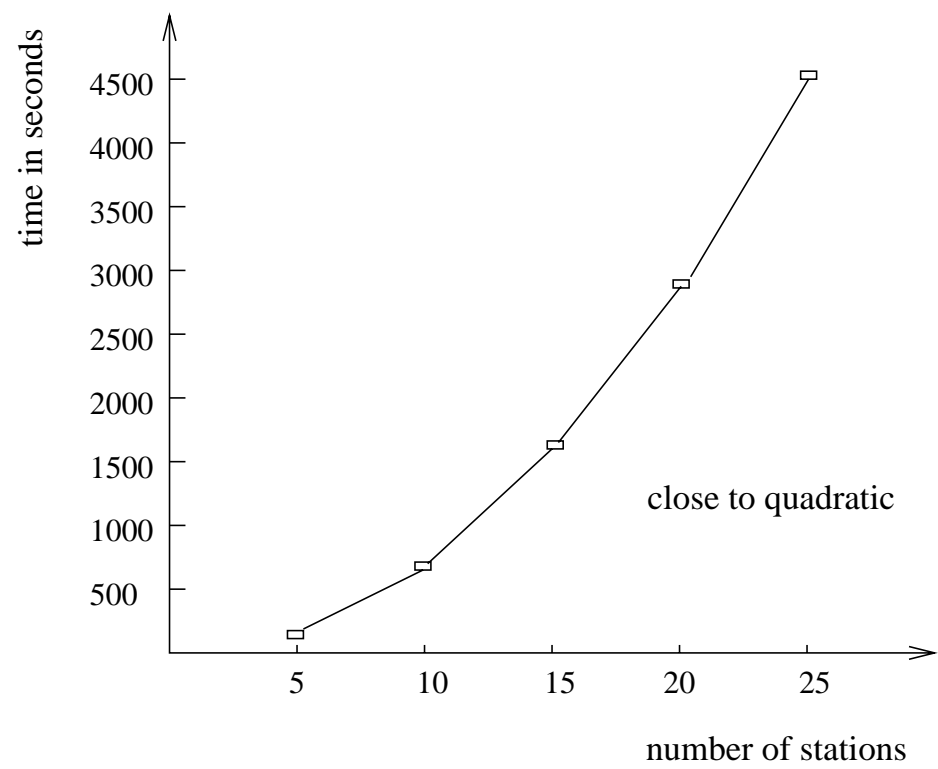

FIG. 5. Computation time versus the number of stations (with precision fixed at 0.0001).

This problem demonstrates that our algorithm can solve rather large problems. It is our experience that $(S C S C L P)$ is easier to approximate than to solve exactly. The computational time grows almost exponentially with the accuracy requirement. A key feature of Algorithm $\mathcal{A}$ is that it keeps the number of breakpoints as small as possible, which in turn makes the size of intermediate quadratic programming subproblems small. It is this feature that makes the algorithm efficient. We believe that Algorithm $\mathcal{A}$ can be made even more efficient if the special structure of the intermediate quadratic programs is exploited.

\section{REFERENCES}

[1] E. J. Anderson, P. Nash, And A. F. Perold, Some properties of a class of continuous linear programs, SIAM J. Control Optim., 21 (1983), pp. 758-765.

[2] E. J. Anderson and P. Nash, Linear Programming in Infinite Dimensional Spaces, WileyInterscience, Chichester, 1987.

[3] E. J. Anderson And A. B. Philpott, A continuous-time network simplex algorithm, Networks, 19 (1989), pp. 395-425.

[4] E. J. Anderson, A Continuous Model for Job-Shop Scheduling, Ph.D. thesis, University of Cambridge, Cambridge, UK, 1978.

[5] K. M. Anstreicher, Generation of Feasible Descent Directions in Continuous Time Linear 
Programming, Tech. report SOL 83-18, Department of Operations Research, Stanford University, Stanford, CA, 1983.

[6] F. Avram, D. Bertsimas, And M. Ricard, Optimization of multiclass queueing networks: A linear control approach, in Stochastic Networks, Proc. IMA, F. Kelly and R. Williams, eds., Springer-Verlag, New York, 1995, pp. 199-234.

[7] R. Bellman, Bottleneck problem and dynamic programming, Proc. Nat. Acad. Sci., 39 (1953), pp. 947-951.

[8] R. Bellman, Dynamic Programming, Princeton University Press, Princeton, NJ, 1957.

[9] D. P. Bertsekas and J. N. Tsitsiklis, Parallel and Distributed Computation: Numerical Methods, Prentice-Hall, Englewood Cliffs, NJ, 1989.

[10] W. P. Drews, A simplex-like algorithm for continuous-time linear optimal control problems, in Optimization Methods for Resource Allocation, R. W. Cottle and J. Krarup, eds., Crane Russak and Co., New York, 1974, pp. 309-322.

[11] J. Eckstein, Splitting Methods for Monotone Operators with Applications to Parallel Optimization, Ph.D. thesis, Massachusetts Institute of Technology, Cambridge, MA, 1989.

[12] J. Filipiak, Modeling and Control of Dynamic Flows in Communication Networks, SpringerVerlag, Berlin, 1988.

[13] R. C. Grinold, Continuous Programming, Ph.D. thesis, Operations Research Center, University of California, Berkeley, CA, 1968.

[14] R. C. GRInold, Continuous programming part one: Linear objectives, J. Math. Anal. Appl., 28 (1969), pp. 32-51.

[15] R. C. GRINOLD, Symmetric duality for continuous linear programs, SIAM J. Appl. Math., 18 (1970), pp. 84-97.

[16] W. W. Hager And S. K. Mitter, Lagrange duality theory for convex control problems, SIAM J. Control Optim., 14 (1976), pp. 843-856.

[17] W. W. HAGER, Lipschitz continuity for constrained processes, SIAM J. Control Optim., 17 (1979), pp. 321-338.

[18] B. HajeK AND R. G. OGIeR, Optimal dynamic routing in communication networks with continuous traffic, Networks, 14 (1984), pp. 457-487.

[19] J. M. HARrison, Brownian Motion and Stochastic Flow Systems, Graduate School of Business, Stanford University, Stanford, CA, Robert E. Krieger Publishing, Malabar, FL, 1990.

[20] J. Hartberger, Representation extended to continuous time, in Optimization Methods for Resource Allocation, R. W. Cottle and J. Krarup, eds., Crane Russak and Co., New York, 1974, pp. 309-322.

[21] A. E. Ilyutovich, Iterative optimization method for linear programming problems in functional spaces, in Studies in Linear and Nonlinear Programming, Stanford University Press, Stanford, CA, 1976.

[22] A. E. Ilyutovich, Piecewise-continuous solutions of linear dynamic problems in economic planning, Automat. Remote Control, 41 (1976), pp. 501-508.

[23] S. Ito, C. T. Kelley, and E. W. Sachs, Inexact primal-dual interior point iteration for linear program in function spaces, Comput. Optim. Appl., 4 (1995), pp. 189-202.

[24] M. Kojima, T. Noma, and A. Yoshise, A Unified Approach to Interior Point Algorithms for Linear Complementarity Problems, Research report RJ 7493, IBM Almaden Research Center, San Jose, CA, 1990.

[25] P. R. Kumar, Re-Entrant Lines, Queueing Systems Theory Appl., 17 (1993), pp. 87-110.

[26] R. S. Lehman, On the Continuous Simplex Method, Tech. report RM-1386, Rand Corporation, Santa Monica, CA, 1954.

[27] N. Levinson, A class of continuous linear programming problems, J. Math. Anal. Appl., 16 (1966), pp. 73-83.

[28] Y. Y. Lin AND J.-S. PANG, Iterative methods for large convex quadratic programs: A survey, SIAM J. Control Optim., 25 (1987), pp. 383-411.

[29] D. G. Luenberger, Optimization by Vector Space Methods, John Wiley, New York, 1969.

[30] D. G. Luenberger, Introduction to Linear and Nonlinear Programming, Addison-Wesley, Reading, MA, 1973.

[31] Z.-Q. LuO AND P. TSENG, Error bound and convergence analysis of matrix splitting algorithms for the affine variational inequality problem, SIAM J. Optim., 2 (1992), pp. 43-54.

[32] X. D. Luo, Continuous Linear Programming: Theory, Algorithms and Applications, Ph.D. thesis, MIT Operations Research Center, Cambridge MA, 1995.

[33] B. Martos, Nonlinear Programming, Theory, and Methods, North-Holland, Amsterdam, 1975.

[34] F. H. Moss And A. Segall, An optimal control approach to dynamic routing in networks, 
IEEE Trans. Automat. Control, AC-27 (1982), pp. 329-339.

[35] F. H. Moss, The Application of Optimal Control Theory to Dynamic Routing in Data Communication Networks, Ph.D. thesis, Department of Aeronautics and Astronautics, Massachusetts Institute of Technology, Cambridge, MA, 1977.

[36] K. G. MurTy, Linear Complementarity, Linear and Nonlinear Programming, Helderman, Verlag, Berlin, 1988.

[37] A. F. Perold, Fundamentals of a Continuous Time Simplex Method, Tech. report Sol 78-26, Department of Operations Research, Stanford University, Stanford, CA, 1978.

[38] A. F. PEROLD, Extreme points and basic feasible solutions in continuous time linear programming, SIAM J. Control Optim., 19 (1981), pp. 52-63.

[39] A. B. Philpott and M. Craddock, An Adaptive Discretization Algorithm for a Class of Continuous Network Programs, Department of Engineering Science, University of Auckland, Auckland, New Zealand, submitted.

[40] M. C. Pullan, A. Mason, M. Craddock, and A.B. Philpott, Computer Programs for Solving the Capacitated Transshipment Problem, School of Engineering Report 521, University of Auckland, Auckland, New Zealand, 1992.

[41] M. C. PUllan, An algorithm for a class of continuous linear programming programs, SIAM J. Control Optim., 31 (1993), pp. 1558-1577.

[42] M. C. Pullan, Forms of optimal solutions for separated continuous linear programs, SIAM J. Control Optim., 33 (1995), pp. 1952-1977.

[43] M. C. PUllan, A duality theory for separated continuous linear programs, SIAM J. Control Optim., 34 (1996), pp. 931-965.

[44] R. G. Segers, A generalized function setting for dynamic optimal control problems, in Optimization Methods for Resource Allocation, R. W. Cottle and J. Krarup, eds., Crane Russak and Co., New York, 1974, pp. 279-296.

[45] S. P. Sethi, W. P. Drews, And R. G. Segers, A unified framework for linear control problems with state-variable inequality constraints, J. Optim. Theory Appl., 36 (1982), pp. 93-109.

[46] W. F. Tyndall, A duality theorem for a class of continuous linear programming problems, SIAM J. Appl. Math., 13 (1965), pp. 644-666.

[47] W. F. TyndalL, An extended duality theorem for continuous linear programming problems, SIAM J. Appl. Math., 15 (1967), pp. 1294-1298.

[48] R. J. VAnderbeI, LOQO User's Manual, Program in Statistics and Operations Research, Princeton University, Princeton, NJ.

[49] G. Weiss, On the optimal draining of re-entrant fluid lines, in Stochastic Networks, Proc. IMA, F. Kelly and R. Williams, eds., Springer-Verlag, New York, 1995.

[50] M. YAMASAKI, Duality theorems in mathematical programmings and their applications, J. Sci. Hiroshima Univ. Ser. A-1, 32 (1969), pp. 331-356.

[51] Y. YE, A fully polynomial-time approximation algorithm for computing a stationary point of the general linear complementarity problem, Math. Oper. Res., 18 (1993), pp. 334-345. 